\title{
Modularity and Hierarchy in Persian Geometric Ornament
}

\author{
Peter R. Cromwell ${ }^{1}$
}

Published online: 7 January 2016

(C) Kim Williams Books, Turin 2016

\begin{abstract}
We survey 40 examples of 2-level patterns in Islamic geometric ornament from Iran and Central Asia, and identify a range of methods that can be used to explain their construction. Techniques based on modular design are shown to simplify the process. Each 2-level pattern is listed in a catalogue that gives the location, construction method, pattern type of the large and small-scale patterns, and references to photographs and other literature. We also show that a tympanum pattern in the Darb-i Imam in Isfahan is constructed by filling compartments and not by subdividing modules, as has been previously suggested. None of the medieval patterns in the catalogue is quasiperiodic.
\end{abstract}

\section{Introduction}

There are many examples of Islamic ornament in which motifs and patterns of different kinds and sizes are combined in an imaginative display of complexity, ingenuity and craftsmanship. These compositions are not formed of independent parts juxtaposed in competition with each other, but are often carefully coordinated so that the different elements support, highlight and complement one another. In this paper we study a particular development of this genre, one in which geometric patterns of two different scales are combined. In the 2-level designs we analyse, a small-scale pattern either fills or outlines the compartments in a large-scale pattern; these two modes (filling and outlining) correspond to Type A and Type B, respectively, in the classification of 2-level designs introduced by Bonner (2003).

Peter R. Cromwell

spmr02@liverpool.ac.uk

1 Pure Mathematics Division, University of Liverpool, Mathematical Sciences Building, Peach Street, Liverpool L69 7ZL, UK 
There is also a third mode, recorded first in the Friday Mosque in Cromwell (2012b), in which the small-scale pattern performs both functions in a single design.

Previous studies of Islamic geometric ornament (Bonner 2003; Cromwell 2009, 2010a, c, 2012a; Lu and Steinhardt 2007) have found evidence to support the traditional use of a modular approach to pattern design. The modules are arranged to form a tessellation, which is either taken as the final design itself or is used as an invisible substrate to layout other motifs. While modular design is not the only method used in the production of Islamic patterns, we shall see that it is one of the fundamental tools for creating 2-level patterns-modules that produce the smallscale pattern are arranged to cover areas or strips that define the large-scale pattern.

The following general principles have been distilled from previous analyses of Persian 2-level patterns (Cromwell 2009, 2010c, 2012a; Cromwell and Beltrami 2011).

- The large-scale pattern is simple and easily recognisable, often an early pattern that is very familiar.

- The relationship between the large- and small-scale patterns is determined by some key points in the large-scale pattern. When the small-scale pattern fills the large-scale compartments, the key points are the corners of the compartments. When the small-scale pattern outlines the large-scale compartments, the key points are the corners of the pathways forming the frames.

- Small-scale stars are centred at the key points of the large-scale pattern. This means focal points in the large- and small-scale patterns coincide. The remaining edge segments of the compartments or pathways are covered by edges or mirror-lines of elements in the small-scale pattern.

- The interior regions of the compartments or pathways are completed in a consistent manner-congruent regions generally have the same filling. The filling need not preserve the symmetry of a compartment.

The same principles have been observed in both the direct tessellation (Cromwell 2012a) and invisible substrate (Cromwell 2010c) forms of modular construction.

This paper presents a survey of methods for constructing 2-level geometric patterns that conform to these principles. It also highlights unusual examples that have non-standard features. All the patterns used to illustrate the techniques are taken from traditional Islamic sources, both buildings and manuscripts, and cover a period of about four hundred years starting in the early fifteenth century. Although many of the architectural examples are located in modern day Iran, the term 'Persian' in the title of the paper refers to the area of historic Persian influence, which extends into Central Asia. I have discussed constructions of 2-level patterns in other papers (Cromwell 2009, 2010b, c, 2012a; Cromwell and Beltrami 2011) and have avoided re-using examples that I have analysed before wherever possible. This paper builds on my previous work but can be read independently.

The appendix contains a catalogue of 40 traditional 2-level geometric patterns. References in the text of the form \# plus a number indicate an entry in the catalogue. The appendix includes descriptions of locations, references to photographs and other literature, summary data about the method of construction and other geometric properties. 
Presenting such a large number of examples together makes it possible to see trends in the development of themes and styles and to compare the geometric examples studied here to the much broader exploration of multiscale ornament. For example, while the bulk of the decoration on the Friday Mosque in Yazd is Timurid in style, the 2-level pattern above the entrance portal has features that do not appear elsewhere until much later. We shall also see that the designs recorded in the Topkap1 Scroll are more advanced than any found in surviving mosaic panels. Finally, in the case of Persian patterns, we can settle the debate about whether medieval mosaics exhibit the mathematical property of quasiperiodicity. When the controversial patterns are placed in the wider context, we see that they are created using the same method as all the others-by filling the large-scale compartments with a small-scale pattern, and not by subdividing large-scale modules as has been proposed (Lu and Steinhardt 2007). Nothing exceptional is required to explain their construction.

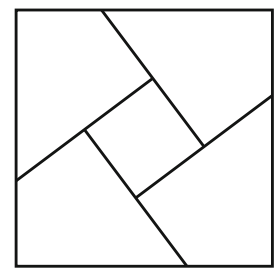

(a)

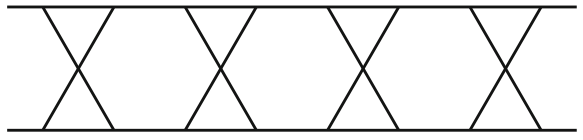

(b)

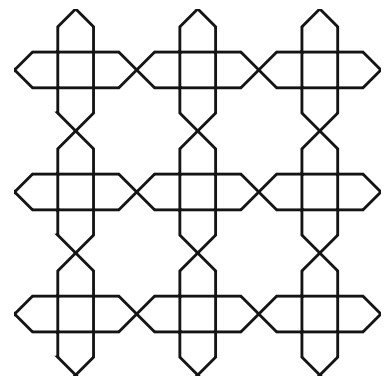

(c)

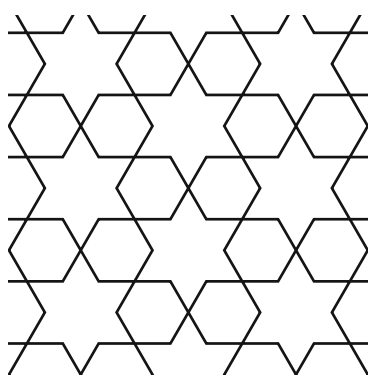

(d)

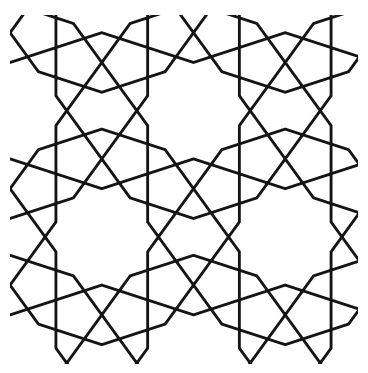

(e)

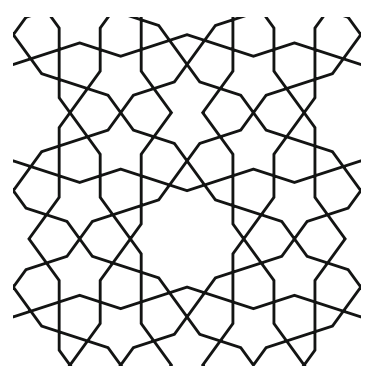

(f)

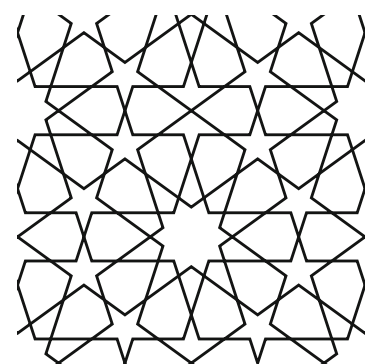

(g)

Fig. 1 Common designs used as large scale patterns 


\section{The Choice of Large-scale Patterns}

The large-scale pattern in a 2-level design provides a framework for the composition. In Persian examples it is usually something simple and easily recognised. This is the case whether the small-scale pattern is composed of geometry, floral or foliate arabesque, or calligraphy. In 29 of the 40 2-level patterns in the catalogue the large-scale pattern is one of the designs shown in Fig. 1. These patterns are archetypal Islamic patterns that are widespread throughout the Islamic world and are very familiar to viewers of Islamic ornament.

The Whirling Kites motif, shown in Fig. 1a, is a square panel divided into four congruent kites chasing around a small central square. The geometry of the motif can be adjusted to produce a larger or smaller square in the centre; the continuously variable family can be parametrised by the small angle in the kites. In the example shown here the long and short sides of the kites are in the ratio 2:1, giving an angle of about $53.13^{\circ}$. The ornamental possibilities of this motif as the large-scale pattern in a 2-level design were explored in Safavid Isfahan-see Cromwell and Beltrami (2011). Its treatments include compartments with geometric filling (\#9, \#22),

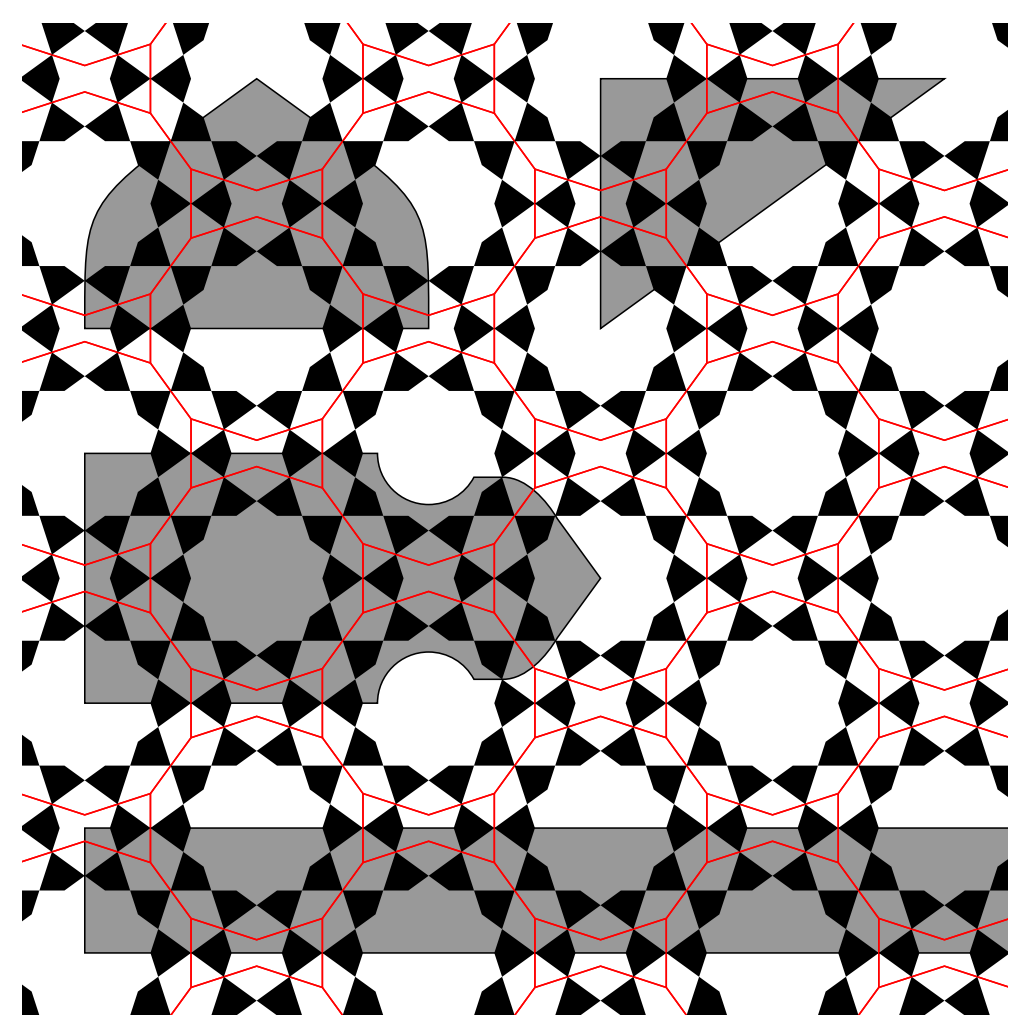

Fig. 2 Subsets of the ubiquitous Umm al-Girih pattern that have been used as the large-scale design in 2-level geometric patterns 
compartments filled with Kufic calligraphy (al-Hakim Mosque, Isfahan-photograph IRA 1017 in Wade 2015), compartments with arabesque filling outlined with geometric pathways (\#7), compartments with Kufic filling outlined with geometric pathways (\#8), and compartments outlined and filled with floral arabesque (Imam Mosque, formerly the Royal Mosque, Isfahan-photograph IRA 0225 in Wade 2015).

Figure $1 \mathrm{~b}$ is a strip pattern composed of hexagons and triangles. In the figure the hexagons are regular, but they may be elongated to provide larger panels, and they need not all be congruent. A sequence of hexagonal panels is sometimes used as an alternative to the simple column of rectangles.

The other five patterns in Fig. 1 are all star patterns. The Star and Cross derivative (c) and the Star and Hexagon pattern (d) are pre-Islamic and can be found in Roman ornament; the others are early Islamic patterns. These simple patterns can be generated in many ways. Probably, the early examples were made by putting stars at the vertices of a standard grid and extending the edges of the stars into the free spaces between: 6-pointed stars on a triangular grid, 8-pointed stars on a square grid, and 10-pointed stars on a rhombic grid. The high density of stars in these patterns is one of the features that makes them recognisable from a small fragment.

The pattern shown in Fig. 1e is very common. According to Sutton (2007, p. 34), it is known in Persian as 'umm al-girih', meaning 'mother of knots'. (Girih is the name for the technique used in Persia and Central Asia to create star patterns- the 'knots' may refer to the vertices in the underlying grids used to lay out the designs.) This ubiquitous pattern is certainly a good candidate to be the 'mother of patterns'. The pattern is redrawn in Fig. 2 using a different style - the kites have been filled in rather than outlined as they are in Fig. 1. The regions highlighted in grey show four excerpts that have been used to provide the large-scale pattern in 2-level geometric designs in a range of architectural features: a tympanum (\#4), a spandrel (\#3), a keyhole arch that forms part of a larger rectangular panel (\#17), and a strip running round a soffit (\#10).

Figure 2 also includes a tiling overlaid on the pattern: the 10-pointed stars have been circumscribed by regular decagons, leaving bow-tie shaped hexagons in the spaces between. The decagon and bow-tie tiles are modules in one of the modular systems we shall meet in the next section. Irregular shapes, such as the bow-tie, that were initially fillers became design elements in their own right. The other star patterns in Fig. 1 can also be constructed with modular systems.

The four grey regions in Fig. 2 illustrate the typical relationship of a panel to a large-scale pattern: the corners of the panel lie at the centres of modules (often at star centres), the straight segments in the panel boundary coincide with mirror lines of modules, and curved segments of the boundary run through stars. In the case of spandrels the pattern may be deformed or adapted slightly to allow for the curvature of the lower edge of the panel. The relationship between compartments and the small-scale pattern follows similar rules. 


\section{Four Modular Design Systems}

The construction of a large variety of different structures from a small set of basic elements (modules) is known as modular design. Modular design enables the construction of patterns with a low density of stars by providing a simple mechanism to produce a matrix of interconnecting shapes. This will be demonstrated in the construction of the small-scale patterns as stars are placed at the focal points of the design and the other shapes are used to link them.

Figures 3, 4, 5 and 6 show modular systems that can be used to create families of geometric patterns found in Islamic ornament. I have named the individual modules for ease of reference (the names are not traditional).

The first modular system, shown in Fig. 3, is a set of polygonal tiles that can be assembled to form tessellations. The dotted lines added to two of the tiles are only to indicate internal structure and do not form part of the final pattern-they show that both tiles can be composed of house modules and small squares. This system, like the others, is sometimes supplemented with other modules (Cromwell 2012a); the modules shown here are those most commonly used.

The three other modular systems work in a slightly different way. Each module is decorated with a motif, shown in black in the figures. The modules are still assembled to form tessellations; the white areas of each module are fused with those of neighbouring modules to form the 'background' regions of the pattern. The module boundaries are not shown in the finished pattern so, whereas the modules in Fig. 3 are directly visible as elements in the composition, the modules in decorated systems provide a hidden substrate to organise the motifs.

Mathematicians denote regular stars by $\{p / q\}$ where $p$ and $q$ are small integers: the star $\{p / q\}$ is formed by placing $p$ points equally spaced around a circle and drawing straight lines that connect points $q$ steps apart. We have denoted each modular system with a symbol of the form $M\{p / q\}$ where $\{p / q\}$ is the star module or star motif that dictates the geometry of the system-it controls the angles in both the tiles and the motifs. In the decorated systems we decorate the $M$ with a tilde: $\widetilde{M}$.

The properties of the plain and decorated modular systems have been described before but we shall review them here.

- The abstraction and recognition of $M\{8 / 2\}$ as a traditional modular system is a recent discovery (Cromwell 2012a). The module shapes (Fig. 3) are derived from the pre-Islamic Star and Cross pattern. The internal angles of the modules are all multiples of $45^{\circ}$ and the edges have two lengths: the long and short edges

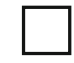

square house

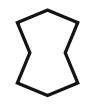

bone

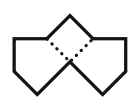

Dutch bonnet

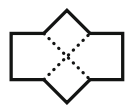

belt

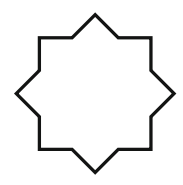

star

Fig. 3 Tiles of the modular system $M\{8 / 2\}$ 


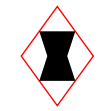

rhombus

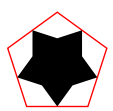

pentagon

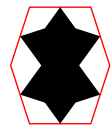

barrel

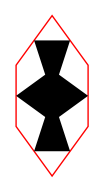

bobbin

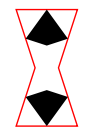

bow-tie

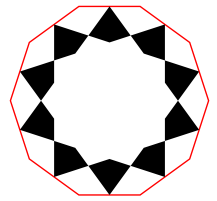

decagon

Fig. 4 Tiles of the decorated modular system $\widetilde{M}\{10 / 3\}$

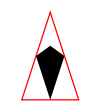

triangle

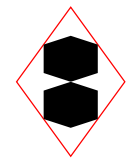

rhombus

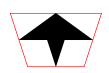

trapezium

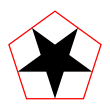

pentagon

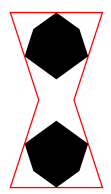

bow-tie

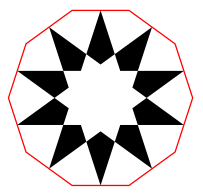

decagon

Fig. 5 Tiles of the decorated modular system $\widetilde{M}\{10 / 4\}$
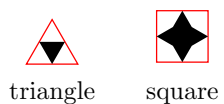

square

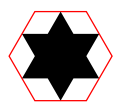

hexagon

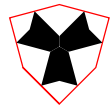

shield

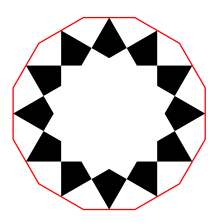

dodecagon

Fig. 6 Tiles of the decorated modular system $\widetilde{M}\{12 / 4\}$

are in the ratio $\sqrt{2}: 1$. Surviving patterns suggest that modular systems are regional. Traditional examples of this one are concentrated in Central AsiaTransoxiana (modern Uzbekistan) and Khorasan (Afghanistan plus areas of neighbouring states, including north-east Iran). Figure 1c can be made with this system.

- The $\widetilde{M}\{10 / 3\}$ modular system has been identified several times and written about quite widely (Cromwell 2009, 2010c; Lu and Steinhardt 2007; Makovicky 1992; Rempel 1961). Bonner (2003) refers to this as the '5-fold system, median family'. The modules (Fig. 4) are equilateral and the internal angles are all multiples of $36^{\circ}$. The motifs are produced by placing two lines at the midpoint of each edge making an angle of $54^{\circ}$ with the edge, and extending them inside the tile until they meet similar lines grown from other edges. The kite motifs on the bow-tie are congruent to those on the decagon. Figure 1e, f can be made with this system.

Some patterns use most or all of the modules, but there are also two subsets of this system in common use. The set \{bobbin, bow-tie, decagon $\}$ is the basis of so many patterns that we shall call it the standard triad. Several of the examples we discuss later use this system. The other subset contains all the tiles that have an internal angle of $108^{\circ}$ (rhombus, pentagon, barrel) plus the decagon. Between 
them, the two sets use all the tiles and only have the decagon in common.

These subsystems exhibit a regional bias in their distribution. Patterns using the standard triad are concentrated in Iran. The other subset seems to be preferred in Transoxiana; it is occasionally supplemented with the distinctive bobbin module. The bow-tie module is rarely found in Central Asia (the umm al-girih pattern is found here as everywhere else, but it can be produced using nonmodular methods). The absence of the bow-tie cannot be explained as a preference for convex modules as $M\{8 / 2\}$ contains non-convex tiles.

- The modules in $\widetilde{M}\{10 / 4\}$ have edges of two different lengths, and the interior angles are all multiples of $36^{\circ}$ (Fig. 5). In this case the long and short edges are in the golden ratio. The pentagon and decagon are regular polygons with short edges, the rhombus and bow-tie are equilateral with long edges, and the two other tiles have both long and short edges. The motifs meet the module boundaries in the midpoints of the edges; the incidence angles are different on the long and short edges. The geometry is dictated by the $\{10 / 4\}$ star on the decagon: it leads to an incidence angle of $72^{\circ}$ on the short edges and $36^{\circ}$ on the long edges. The motif on the pentagon is the $\{5 / 2\}$ star. A strict application of the motif-generation procedure to the rhombus tile produces a long convex hexagon of little aesthetic value; the motif shown in the figure is one of several variants used in practice. Figure $1 \mathrm{~g}$ can be made with this system.

The rhombus and bow-tie modules (the two equilateral tiles with long edges) are uncommon. Patterns built from the four other modules are widely distributed throughout the Islamic world from India through Central Asia and Iran to Turkey and north Africa. The popularity of these four modules may be due to their ease of construction (the trapezium and triangle tiles are simple fragments of the pentagon and decagon tiles, respectively) and the fact that two of the motifs are regular stars. This four-module subset is shown in Hankin (1925, Fig. 33) and Rempel (1961, Fig. 191), and is the core of Bonner's '5fold system, acute family'. It is also logically equivalent to a set of only two decorated rhombic modules discovered by Castéra (2011), who gives a very elegant method for generating the same patterns.

Patterns that use the bow-tie module can be found in Iran, Egypt and Morocco; examples are shown by Bonner (2003, Fig. 19) (the same as our Fig. 14a) and in Cromwell (2012a, Figs. 8 and 9). The rhombus module has been used to explain the structure of patterns found in Spain and Morocco (Cromwell 2009, Fig. 19); however, many patterns in these countries come from the zellij tradition (Castéra 1996) and may be constructed using other methods.

- Of the four systems, the modules in $\widetilde{M}\{12 / 4\}$ are the simplest to constructfour of the five tiles are regular polygons, and the shield tile is formed by attaching three isosceles right triangles around an equilateral triangle (Fig. 6). The tiles are equilateral, their internal angles are all multiples of $30^{\circ}$, and the motifs have an incidence angle of $60^{\circ}$ with the edges. Unfortunately, this ease of construction makes it difficult to establish the traditional use of these tiles for modular design, as many of the patterns they generate can also be produced in other ways. The shield tile is the best evidence of a modular approach, but it 
appears in only a few patterns from Cairo and Damascus. Figure $1 \mathrm{~d}$ can be made with this system.

The modules are shown in Sutton (2007, p. 18), and Plates 92-94 in Bourgoin (1879) provide example patterns. I am not aware of any medieval 2-level patterns that use this system, although it has been employed as the basis for more recent 2-level designs: one in bright modern colours can be found in the Friday Mosque of Gawhar Shad in Mashhad and one by Bonner is on his website (Bonner 2015). We will not consider this system further in this article.

Modular systems lead naturally to structural hierarchies, as the basic modules are grouped together to form larger units. Figure $7 \mathrm{~b}$, c show two patches of tiles from

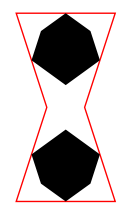

(a)

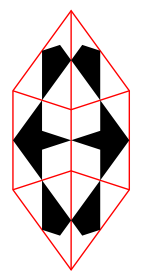

(b)

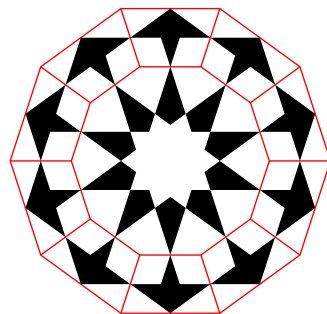

(c)

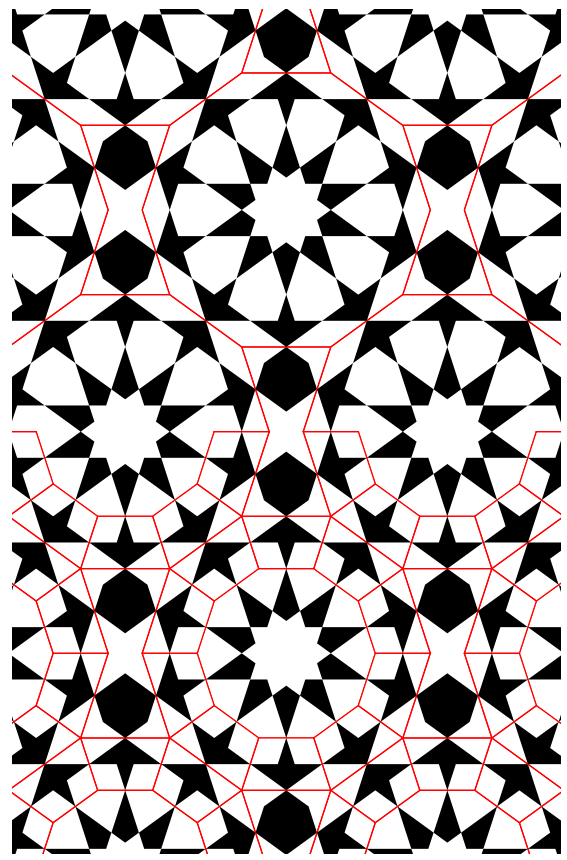

(d) B187a

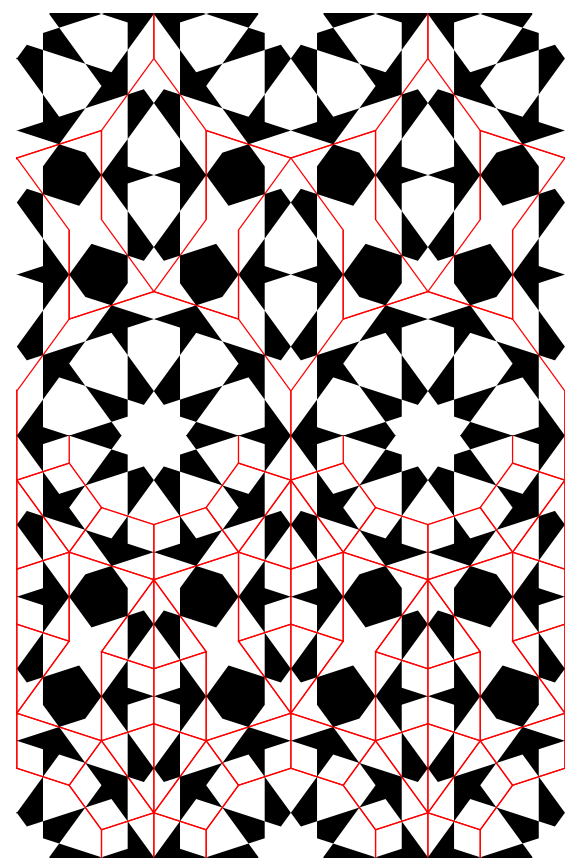

(e) B187b

Fig. 7 A simple hierarchy: patches of tiles from the set $\widetilde{M}\{10 / 4\}$ that imitate the standard triad in $\widetilde{M}\{10 / 3\}$ 
set $\widetilde{M}\{10 / 4\}$. The composite shapes belong to the set $\widetilde{M}\{10 / 3\}$; together with the bow-tie from $\widetilde{M}\{10 / 4\}$ they form a standard triad. Figure 7 d, e show two patterns from Bourgoin's Plate 187 (Bourgoin 1879). The lower part of each pattern is overlaid with the primitive tiles and the upper part with the composite tiles. This simple mechanism of assembling modules into familiar shapes can produce many and complex variations of traditional patterns. We shall see this approach many times in the following examples: we shall use modules to tile pathways that outline compartments, fill the compartments and, as in this example, create larger versions of the modules themselves.

\section{Outlining Compartments}

We now turn to explore methods that can be used to construct 2-level patterns. We start in this section with three methods to produce frames around compartments: splicing sections of strip patterns, leaving gaps in plane patterns, and constructing purpose-made tessellations along the pathways.

\section{Mitred Ribbons}

Perhaps the simplest way to produce a border around a compartment is to take a strip pattern and run it along the boundary, slicing it at the corners and junctions. Where this approach is used in Roman mosaics, there is often no attempt to match the pattern across the joins. In Islamic examples the joins are mitred, which produces a tidy design without loose ends.

Figure 8c shows an example taken from a ceiling in the Balyand Mosque, Bukhara (\#12). It is notable as one of the few 2-level patterns not executed in cut tile mosaic. Instead, the wooden ceiling is suspended from the beams above and the design is applied using kundal: paint and gold leaf on papier maché relief. More examples of this medium can be found in the interiors of other mosques in Bukhara and Samarqand.

The small-scale pattern is formed of tiles from the $M\{8 / 2\}$ modular system. Figure 8a shows four strips of the pattern in Fig. 1c that have been mitred and joined to form a design element in which two paths cross at an angle of $45^{\circ}$. Each strip is bordered by halves of $\{8 / 2\}$ stars, and the crosses running along the centre of a strip have been subdivided into four house modules surrounding a central square. The vertical and horizontal lines radiating from the centre of the figure show where the top-right strip has been cut in the mitring. The choice of the $45^{\circ}$ angle means that the shapes produced by fusing the mitred stars are also fragments of regular $\{8 / 2\}$ stars.

The large-scale pattern is the 12-pointed rose shown in Fig. $8 \mathrm{~b}$. The rose construction is based on tangent circles (shown in the upper half of the figure) and follows (Lee 1987). At each point where two circles meet, the lines of the rose motif cross at an angle of $45^{\circ}$; this requirement suffices to define the geometry of the motif completely. Note that the 12-pointed star in the central circle is not one of the 


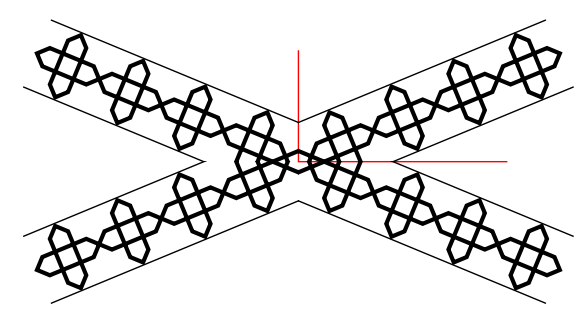

(a)

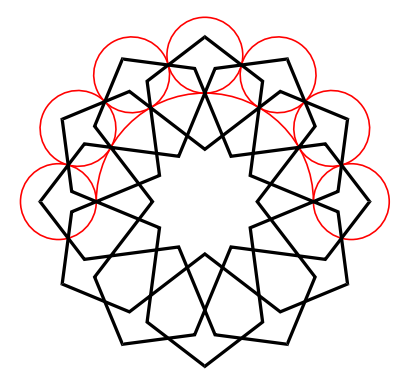

(b)

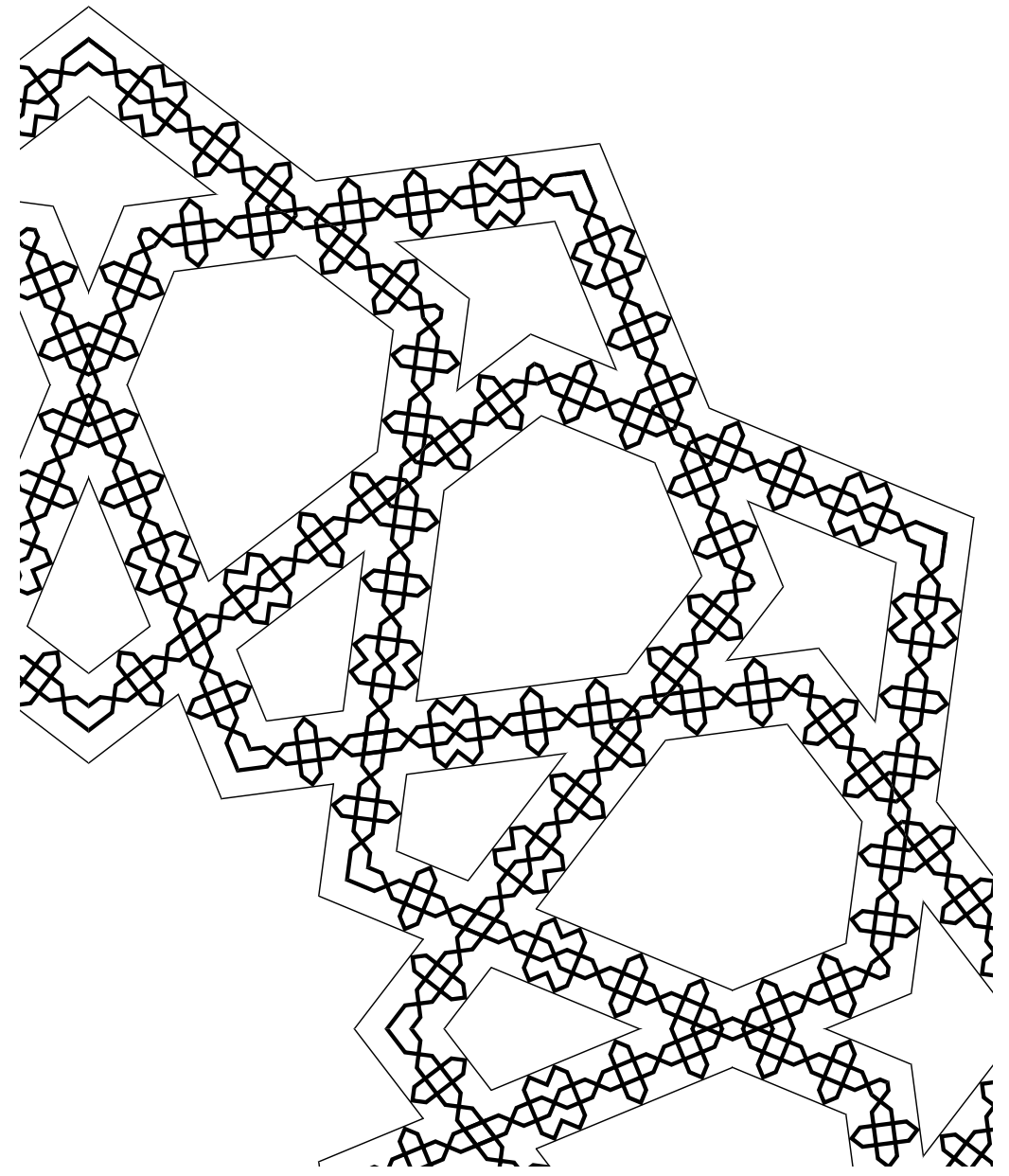

(c)

Fig. 8 Design from a ceiling in the Balyand Mosque, Bukhara (early sixteenth century) 
regular star polygons: in $\{12 / 4\}$ the spikes have an apex angle of $60^{\circ}$ and in $\{12 / 5\}$ the angle is $30^{\circ}$.

These two design elements come together in Fig. 8c. The small-scale pattern decorating the crossed pathways in (a) is used at the $45^{\circ}$ crossings in (b), and the strips are extended along the pathways using other modules from $M\{8 / 2\}$. The angle between the pathways at the inner crossings of the star is $75^{\circ}$, and the normal right angled cross composed of a square and four houses is adapted to fit this skew setting.

At the corners of the rose the small-scale pattern is mitred. This entails fusing fragments of small-scale stars but, because the angles at the corners of the rose are not multiples of $45^{\circ}$, the resulting shape is not part of an $\{8 / 2\}$ star, even when the constituent parts are concentric. The mitring also produces other small-scale shapes that do not belong to the modular system. The production of these spurious shapes is an indicator of this technique, and also its biggest disadvantage.

In other work (Cromwell 2012a, Fig. 15) I have analysed a 2-level design from the soffit of the south iwan of the Friday Mosque of Gawhar Shad in Mashhad (\#35). It dates from 1416-1418, making it one of the earliest 2-level patterns. The smallscale pattern has a similar structure to the Balyand example and is formed of mitred strips of $M\{8 / 2\}$ modules. The large-scale pattern is simpler: it is a field pattern (contains no stars), its lines always cross at right angles, and the angles at the corners are $135^{\circ}$. Just as in the Balyand example, the mitring at the corners produces shapes foreign to $M\{8 / 2\}$ as pairs of overlapping stars or overlapping houses are fused. The mitring is visible in close-up photographs (Golombek and Wilber 1988, Fig. 249) - the $\{8 / 2\}$ star fragments at the corners of the compartments are not whole tiles but are cut along the mitre line, suggesting that the panel has been made in sections corresponding to the strips.

These two patterns are the only Persian examples I know of this technique and it happens that they both use strip patterns composed of modules from $M\{8 / 2\}$. In principle, any strip pattern could be used as the basis of this technique; the use of a modular system may make it easier to align the small-scale pattern to the key points of the pathways as far as possible.

\section{Leaving Holes}

Another simple technique to create framed compartments is to cut holes in a plane pattern. Our examples are all constructed on a grid of equilateral triangles. The plane pattern provides the small-scale pattern and the large-scale pattern is formed by omitting some of the triangular repeat units to create apertures for the compartments.

Figure 9 shows the four star patterns that form the small-scale patterns in our examples of this technique. In each case, the underlying triangular grid is shown by the broken lines. The patterns contain various combinations of stars: (a) has regular $\{12 / 4\}$ stars centred at the vertices of the grid, (b) has 12-pointed stars at the vertices and 9-pointed stars inside the triangles, (c) has $\{6 / 2\}$ stars at the vertices and $\{9 / 3\}$ stars in the triangles, and (d) has $\{12 / 4\}$ stars at vertices and $\{6 / 2\}$ stars 


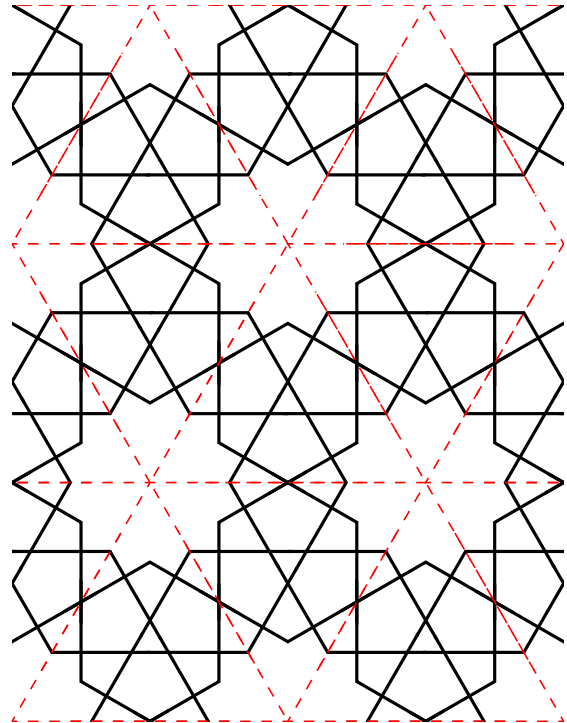

(a)

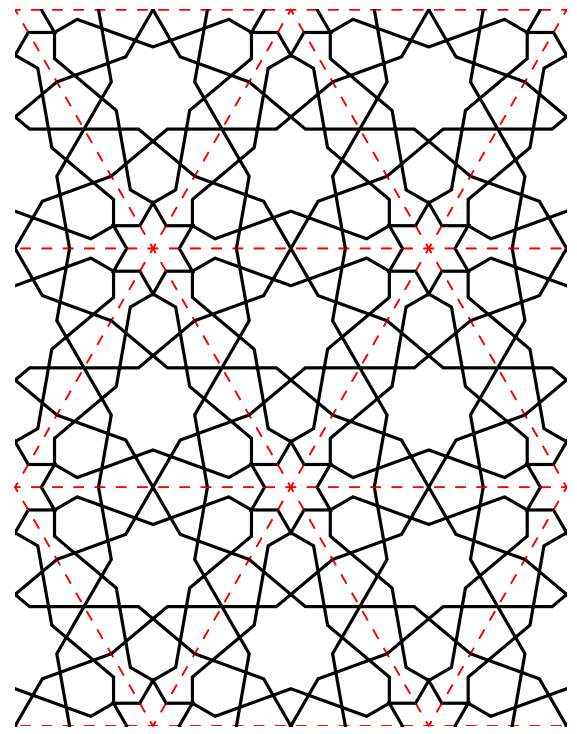

(c)

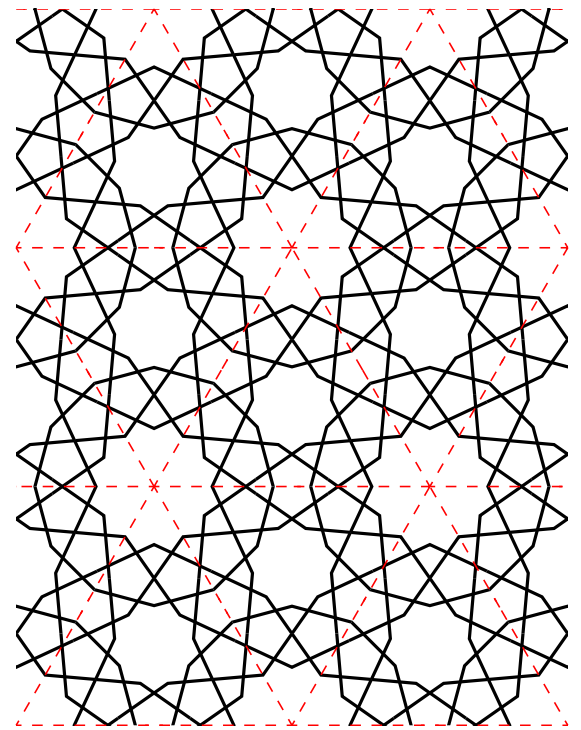

(b)

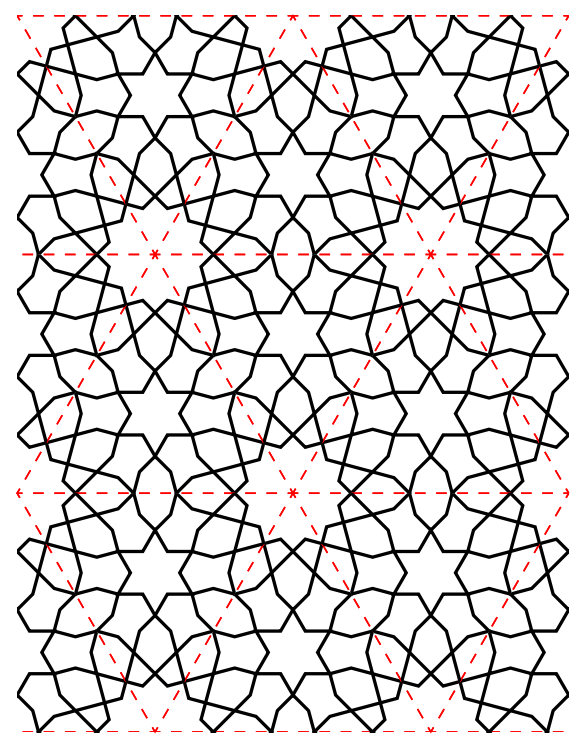

(d)

Fig. 9 Four patterns with triangular repeat units

in the triangles. With the exception of (a), which can be assembled using the triangle and dodecagon modules from $\widetilde{M}\{12 / 4\}$, these patterns are not created with modular systems. The methods of construction do not concern us here-all that matters is the underlying triangular structure. 


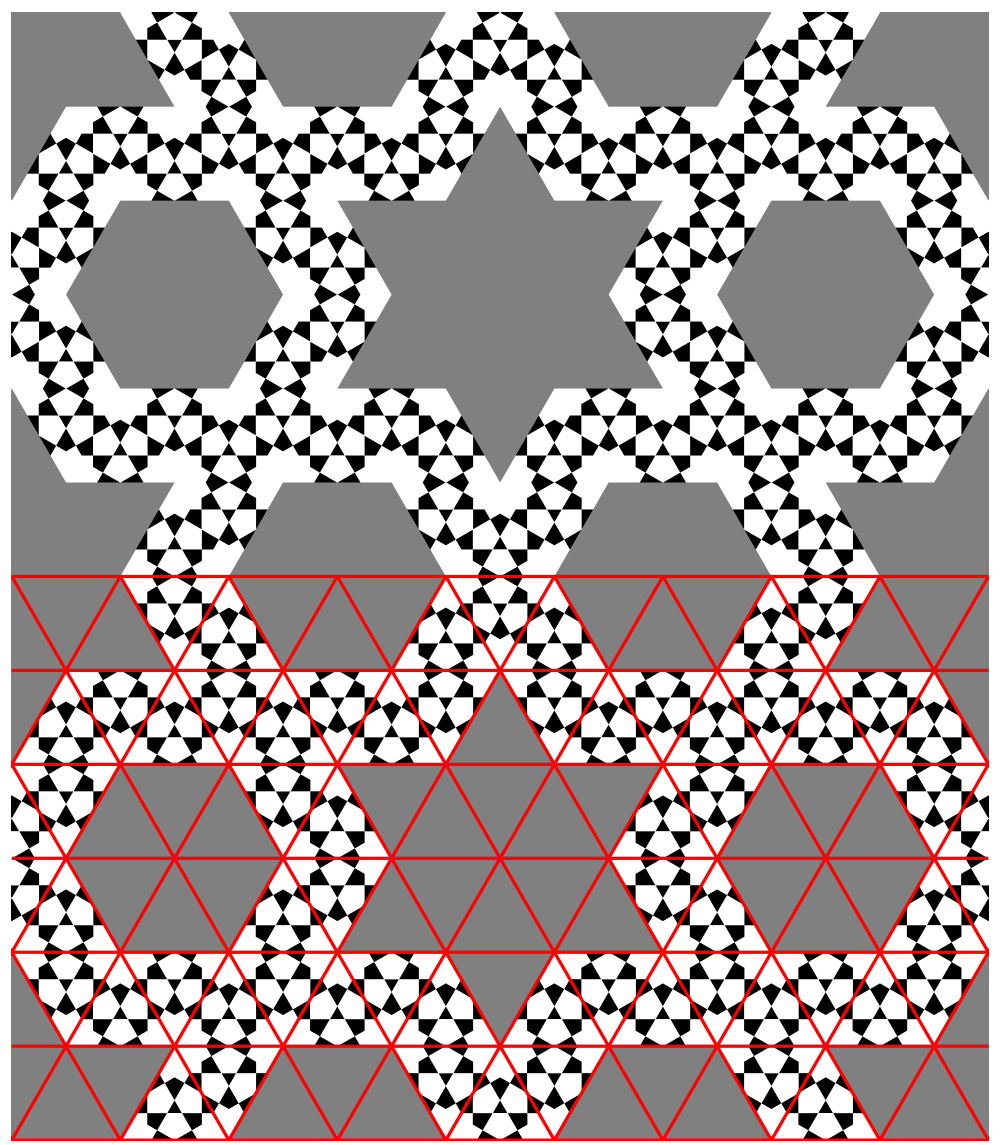

Fig. 10 Design from a relief panel in the Friday Mosque, Varzaneh (1442-1444)

Figure 10 shows an early 2-level pattern. It is from a panel in the Friday Mosque at Varzaneh (\#1) and dates from 1442-1444. The panel is made in relief: the compartments (shown in grey in the figure) are raised up and carry arabesques and Kufic calligraphy, while the small-scale star pattern runs in the channels in between. A flat panel (\#36) in the Darb-i Kushk at Isfahan (1496/1497) is based on the same design, but the craftsmanship is of lower quality. The triangular grid is overlaid on the bottom half of Fig. 10, the small-scale pattern is that of Fig. 9a and the largescale pattern is the pre-Islamic Star and Hexagon design of Fig. 1d. Notice that all the corners of the compartments coincide with the centres of stars in the small-scale pattern. This will be a consistent theme throughout.

The Varzaneh Mosque contains another 2-level pattern (\#2) with the same Star and Hexagon large-scale pattern. In this case Fig. $9 \mathrm{~d}$ is used as the small-scale pattern. The panel is a cut-tile mosaic in black, white, turquoise and dark blue.

The same principle is used to generate some 2-level patterns in Bukhara, Uzbekistan (\#13, \#14, \#15). The large-scale pattern in these examples is a strip of 


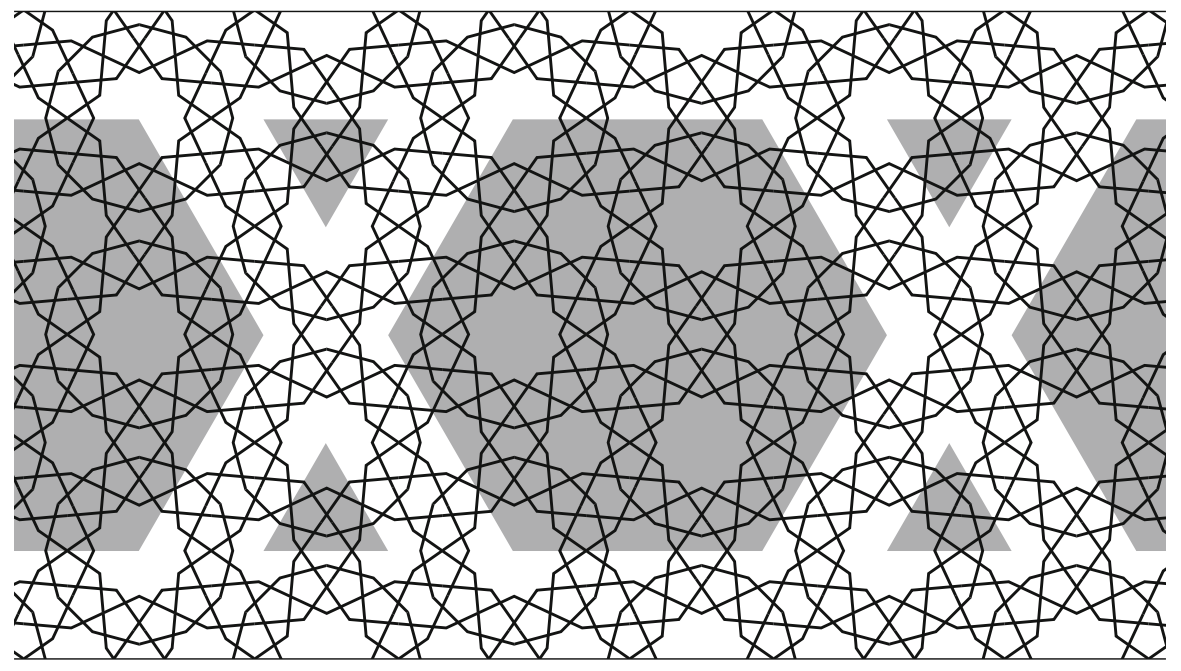

Fig. 11 Design from the ruins of an unidentified madrasa, Bukhara

hexagons and triangles, as shown in Fig. 1b. In the figure the hexagons are regular, but elongated hexagons are also found in some panels. The patterns are applied to the front of large portals to form a frame up the two sides and across the top. Figure 9c provides the small-scale pattern at the Kalyan Mosque (1514) and the Nadir Divan-Begi Madrasa (1622/1623)_\#13 and \#14 in the catalogue, respectively.

A more unusual example (\#15) is shown in photographs TRA 1108, TRA 1120 and TRA 1122 of Wade's collection (Wade 2015) and in Fig. 11. Notice how the alignment of the small-scale and large-scale patterns varies along the strip as the corners of the compartments sometimes lie in 12-pointed stars and sometimes in 9pointed stars. The small-scale pattern is the star pattern of Fig. 9b, but it is not applied at the normal scale. The triangles shown in Fig. 9b connect the centres of the 12-pointed stars. However, the 6-fold and 3-fold rotation centres (situated at the centres of 12-pointed and 9-pointed stars) also form a triangular grid, and it is this finer grid that regulates the large-scale pattern. The scale factor between the two grids is $\sqrt{3}$.

The emergence of 2-level geometric patterns is correlated with advances in technology that produced coloured glazes. The decoration of the Friday Mosque in Varamin (1326), which dates from the Ilkhanid period, consists of stucco and brickwork patterns, some of which contain touches of colour in turquoise and dark blue glaze. These highlights are applied as inserts or coloured strips. Later, glaze became less expensive and available in a wider range of colours, which enabled the complete coverage of large panels and continuous revetments on Timurid buildings.

It is interesting that the ideas needed to produce Fig. 11 are already apparent in Varamin. Photographs IRA 2905-2907 and IRA 2924 from Wade's collection show the lower and upper sections from the soffit of the sanctuary iwan. The triangular 
and hexagonal panels are arranged along the strip as in Fig. 1b and are outlined in simple relief brickwork. The large panels are filled with the triangle-based star patterns of Fig. 9a, b. At Varamin the panels are filled and at Bukhara the panels are outlined, yet the geometry involved in both cases is very similar.

Note that there is nothing difficult about using this technique to generate 2-level designs from known patterns. Figure 9a is a very early Islamic pattern-it appears in the brickwork patterns above the door of the earlier (East) tomb tower at Kharraqan, Iran (1067/1068). Anyone who can construct this star pattern can also construct the 2-level pattern of Fig. 10 simply by omitting some of the repeat units. However, realising the idea in applied ornament requires a medium capable of expressing sharp, fine detail. This need was satisfied by the mass production of glazed tiles in a variety of colours.

It is odd that, although coloured inks also provide a suitable medium for intricate geometric ornament (as is vividly demonstrated by Celtic illuminated manuscripts such as the Book of Kells or the Lindisfarne Gospels), 2-level Islamic patterns are not found in illuminated Qurans.

\section{Modular Filling of Templates}

The method described in the preceding section was presented as taking a continuous pattern and leaving holes in it to make space for the compartments. But it can also be thought of as arranging copies of a triangular template to cover pathways outlining the compartments-a viewpoint that is easy to generalise to other templates. Indeed, new templates can be purpose-made to fit a given large-scale design.

The process is illustrated in Fig. 12. The grey areas in (a) and (b) are templates that we shall use to pave the large-scale pattern. In each case the template is covered with modules from $M\{8 / 2\}$ : a star is placed at each corner, and modules that lie along an edge of the template are placed symmetrically so that a mirror line of the module coincides with the edge of the template. Whole modules are shown in the figure, extending beyond the edges of the template, but they are cropped to fit. The constraints controlling the placement of the modules around the boundary of a template ensure that the small-scale pattern continues naturally across the joins when the templates are assembled.

Figure 12c shows how the templates are arranged to create pathways outlining compartments. The bottom third of the figure shows the template boundaries, the middle third shows the boundaries and the modules, and the top third shows only the small-scale pattern.

This pattern runs around the soffit of the arch of the grand iwan of the shrine of Sheikh Ahmad-i Jam in Turbat-i Jam (\#37). The shrine was built in the fourteenth century but the iwan was redecorated in the Safavid period and the tilework dates from 1613/1614. The pattern is much earlier. A small panel in the Friday Mosque in Isfahan (\#6) dating from 1475/1476 contains the same pattern rotated by $45^{\circ}$. Both the early panel and the later soffit are made in relief with the compartments standing out from the small-scale pattern. 


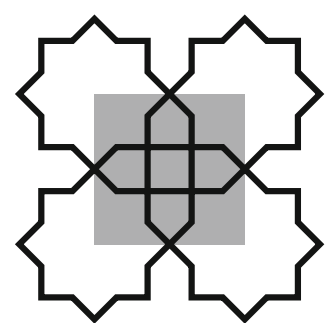

(a)

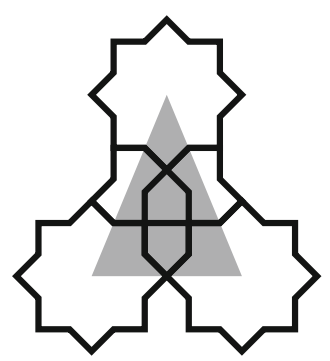

(b)

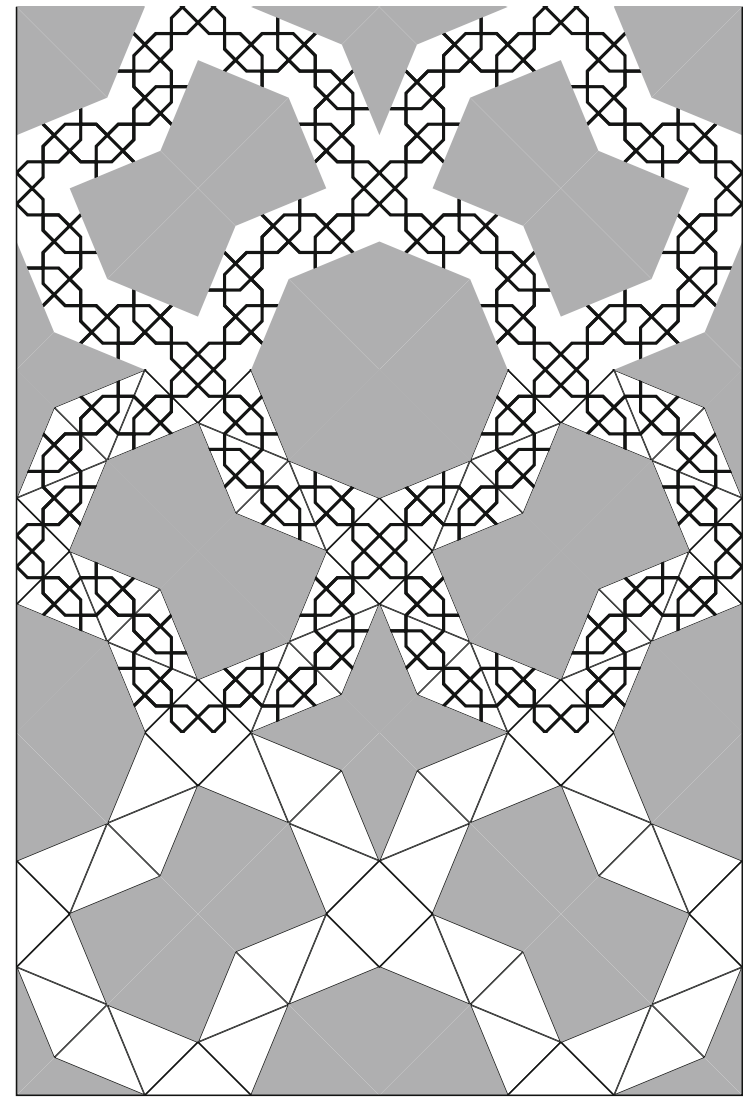

(c)

Fig. 12 Design from the soffit of the grand iwan of the shrine of Sheikh Ahmad-i Jam, Turbat-i Jam $(1613 / 1614)$

This pattern has no mitres, so no fused tiles or other spurious shapes have been created in the small-scale pattern; all the shapes are whole modules or fragments of modules that have been cut along mirror lines. There is also a unity of approach in the placement of the small-scale stars-all the corners of the compartments lie at the centre of a small star. Yet the result is not entirely satisfying.

In the Varzaneh pattern of Fig. 10 the pathways continue straight across at the crossings, and the centre-lines of the pathways form a skeleton for the large-scale pattern. These properties make the large-scale pattern easy to abstract and recognise. Figure 13a reproduces the structure of the Turbat-i Jam pattern and shows that it does not have either of these properties. The thick lines overlaid on the top-right of the figure outline the equilateral polygons that form the skeleton of the large-scale pattern. The individual shapes are familiar-regular octagon, bone and a 4-pointed star-but the skeleton does not lie on the centre-line of the pathways. This offcentre placement, together with the deviation from a straight course across the 


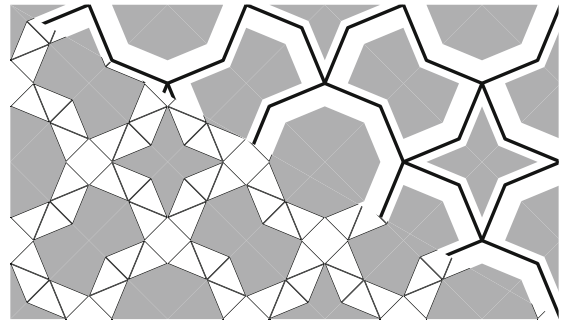

(a)

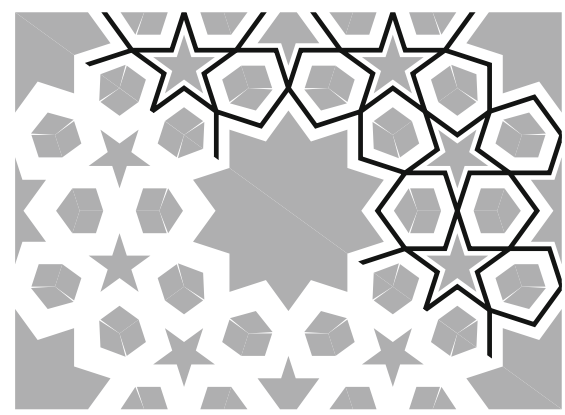

(c)

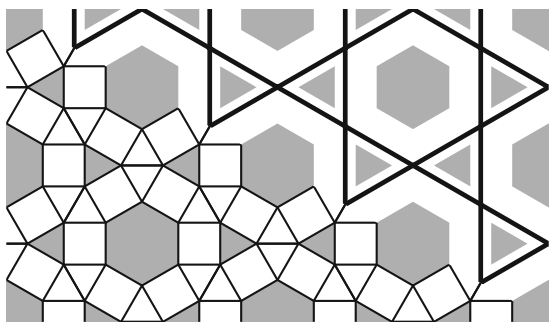

(b)

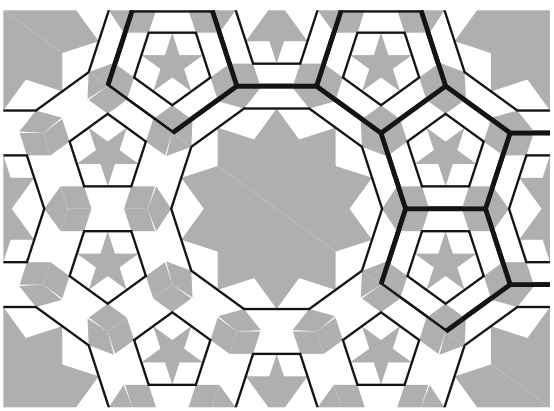

(d)

Fig. 13 In these examples the paths are off-centre from the skeleton

crossings, gives the impression that the paths are not properly aligned and that the large-scale pattern is awkward and unnatural. The pathways feel more like a filler that separates the compartments than a structural framework that is organising the space.

Figure 13 shows two more examples of 2-level patterns in which the pathways are off-centre from the skeleton of the large-scale pattern. Figure $13 \mathrm{~b}$ is taken from panel 38 of the Topkap1 Scroll (\#28) and exists only as a design on paper, albeit a colourful and complete one. The structure of the panel is based on a tiling of regular polygons-triangles, squares and hexagons. The pathways are composed of triangles and squares. In this case, the templates for the small-scale pattern are taken from two existing patterns, one with triangular and one with square repeat units. See Cromwell (2010b) for details of the construction.

Figure $13 \mathrm{c}$ is another early relief panel (\#5); it is from the fifteenth-century Darbi Imam in Isfahan. As in the Friday Mosque panel (\#6) that is the model for the Turbat-i Jam pattern, there is an unresolved tension created by the juxtaposition of conflicting elements. In this case, the 10-pointed star is $\{10 / 3\}$ with apex angles of $54^{\circ}$, the 5-pointed star is $\{5 / 2\}$ with apex angles of $36^{\circ}$, and the irregular 6-pointed shape has angles of different sizes. This causes the paths to deviate at crossings. Furthermore, the skeleton does not follow the centre-lines of the pathways.

There is a more subtle organising principle to this large-scale pattern. Figure $13 \mathrm{~d}$ shows the same design overlaid with a structure formed by connecting corners of the compartments. The thick lines in the top-right of the figure form the familiar tiling 
of regular decagons, regular pentagons, and irregular convex hexagons. This skeleton does lie on the centre-lines of the paths outlined by the overlay. However, none of this framework is apparent to the viewer of the finished mosaic-it is very difficult to find, even when you know where to look.

It is possible to create 2-level patterns in which the large-scale pattern is a complex Islamic star pattern and so that the pathways are centred on the skeleton. Figure 14 shows an example taken from panel 49 of the Topkap1 Scroll (\#29). The large-scale pattern is shown in (a); it is a star pattern that can be constructed with the

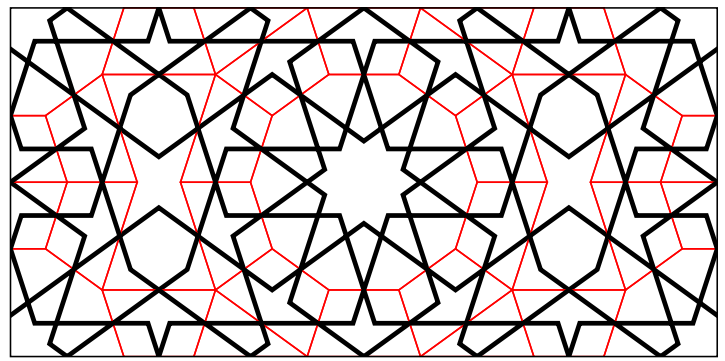

(a)

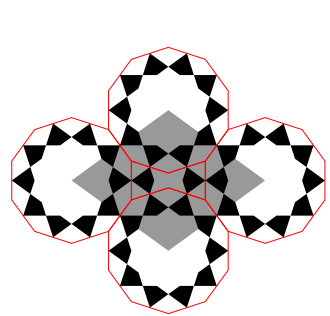

(b)

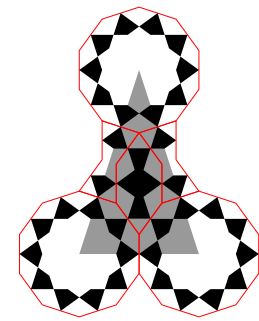

(c)

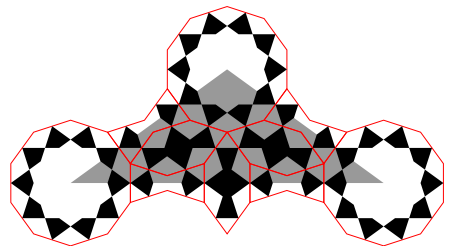

(d)

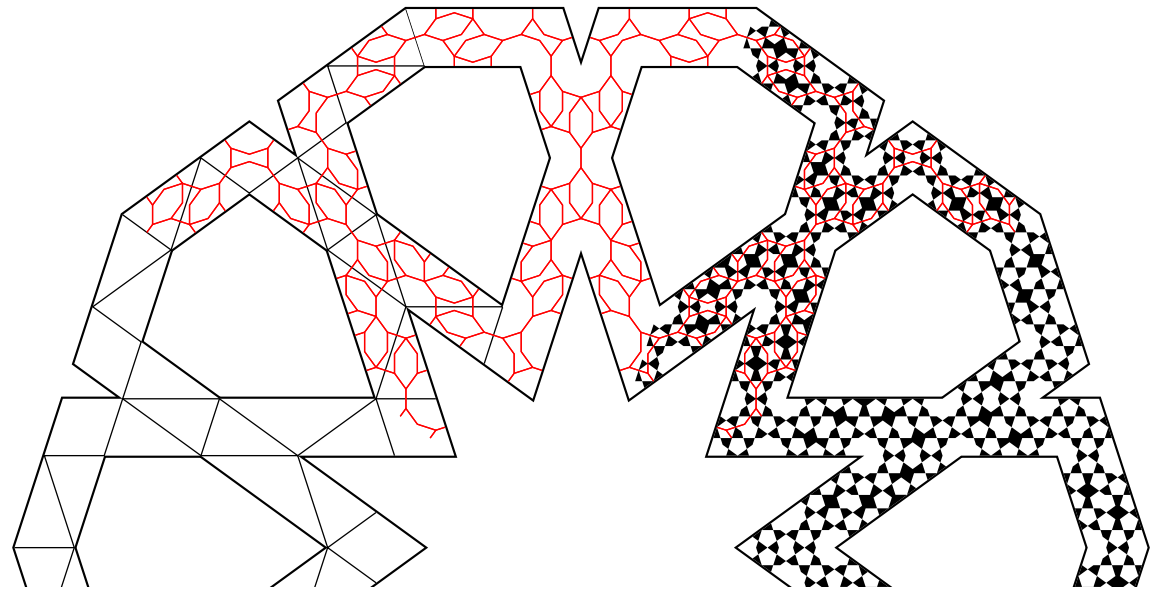

(e)

Fig. 14 Design element from panel 49 of the Topkap1 Scroll 
$\widetilde{M}\{10 / 4\}$ modular system, as shown by the overlaid tiling. The three grey regions in (b)-(d) are some of the templates used to pave the large-scale pattern-a rhombus and two isosceles triangles. Each template is covered with modules from the standard triad in $\widetilde{M}\{10 / 3\}$. As in Fig. 12, stars are placed at the corners of each template, and template boundaries lie on mirror lines of modules.

Figure $14 \mathrm{e}$ illustrates the construction process on a detail extracted from the large-scale pattern - the central rose around a $\{10 / 4\}$ star. It shows stages in the application of the templates to produce the small-scale pattern. Proceeding clockwise, the sectors show (1) the arrangement of templates on the paths, (2) the templates with modules, (3) the module boundaries, (4) the modules decorated with their motifs, and (5) the small-scale pattern. As we noted in the earlier examples, all the corners of the compartments are located in the centres of small stars, and the edges of the pathways lie on mirror lines of the modules. Covering the whole pattern requires two additional templates, but the principle is the same. The details can be found in (Bonner 2003). In her book on the Topkap1 Scroll (Necipoğlu 1995) Necipoğlu annotates the panels to show the indentations and lines scored in the paper that were used in the construction process but which are not inked in and so are not visible to the eye. The annotations for panel 49 show the outlines of the $\widetilde{M}\{10 / 3\}$ modules, supporting this analysis of the small-scale pattern as a modular design.

The pattern in panel 49 of the Topkap1 Scroll proves that, by the time the scroll was compiled, Islamic craftsmen were capable of producing complex 2-level designs with well-aligned pathways and in which the large- and small-scale patterns are perfectly matched with one another. Like the design in panel 38 that we saw earlier, it exists only on paper. I am not aware of any architectural examples of this quality where compartments are outlined with small-scale geometric patterns. We have discussed the Timurid examples from the soffit at Mashhad (\#35) and three panels designed by Sayyid Mahmud (Bonner 2003) at Varzaneh (\#1) and Isfahan (\#5 and \#6). From the later Safavid period we have the soffit at Turbat-i Jam (\#37) based on \#6 and also two Whirling Kites patterns (Cromwell and Beltrami 2011) in the Friday Mosque in Isfahan (\#8 and \#7). There is also a Whirling Kites pattern from the Blue Mosque in Tabriz (\#40) in which the central square is the only compartment whose corners do not coincide with small-scale stars. None of these examples has the perfect geometry displayed in panel 49. However, panel 49 is not a one-off - the capability is combined with the method to be described in the next section to produce patterns in which the outlining and filling of compartments are part of one continuous carpet of ornament.

\section{Filling Compartments}

We now switch our attention from framing compartments to filling them. Here the modular method greatly simplifies the task, almost to the extent that the results become formulaic and routine to produce. All the surviving mosaics seem to be produced by filling the compartments directly, but there is some evidence from the 
Topkap1 Scroll that people had also started to explore the possibility of filling the large-scale modules, leading indirectly to a large-scale pattern covering the compartments. Finally, we shall see the difficulties that arise and the ingenuity required when modular methods are not used.

\section{Revealing the Large-Scale Pattern}

When the small-scale pattern outlines compartments, the large-scale pattern is clearly visible as the structural framework of pathways organising the space. However, when the small-scale pattern fills all the compartments, it is continuous across the whole design and the large-scale pattern must be highlighted explicitly in some way.

Figure 15 shows three ways that the large-scale pattern can be displayed. In (a) a thin line is inlaid along the edges of the large-scale pattern. This is the technique used in the Timurid examples in Isfahan in the Darb-i Imam (\#3 and \#4) and the Friday Mosque (\#10 and \#11). The technique shown in (b) was used in the later Safavid period: the large-scale pattern is laid out in two pale colours such as white and orange-yellow, and the foreground motifs of the small-scale pattern are overlaid in black, leaving the colours of the large-scale pattern to show through in the other areas. In the third method, shown in (c), the large-scale pattern determines the light/dark shading of the small-scale pattern: in some compartments we get dark motifs on a light ground, and in the neighbouring compartments we get light motifs on a dark ground. The light/dark reversal is often accompanied by a colour change. The example shown in the figure comes from the soffit of an iwan in the Vakil Mosque (Regent's Mosque) in Shiraz (\#39); in the large-scale kites the small-scale pattern is executed in yellow on a dark green ground, while in the other regions it has black motifs on a white ground.

\section{Modular Filling of Compartments}

The process of filling compartments is similar to the process of filling the templates that we used in Sect. 4.3 for outlining the compartments. It is illustrated in Fig. 16

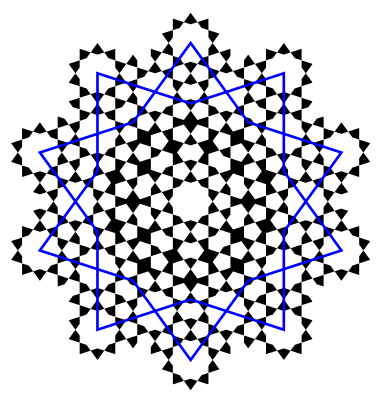

(a) inlaid line

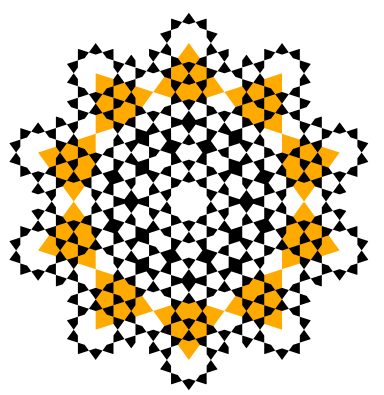

(b) transparent overlay

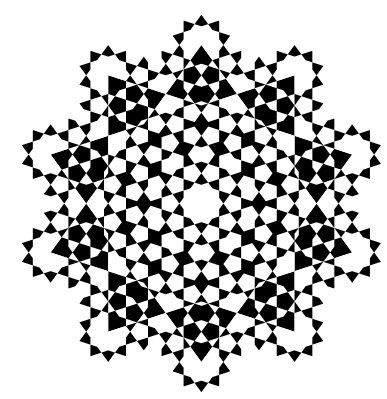

(c) contrast reversal

Fig. 15 Different techniques for displaying the large-scale pattern 


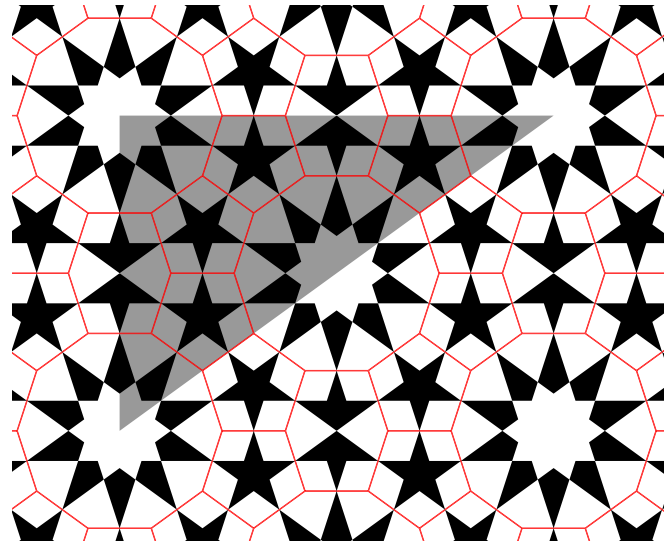

(a)

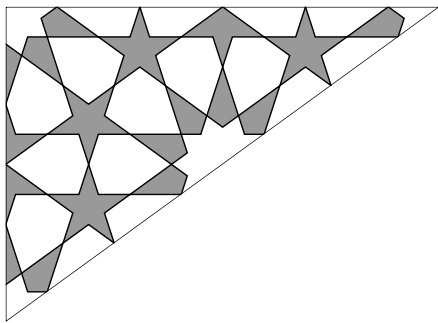

(b)

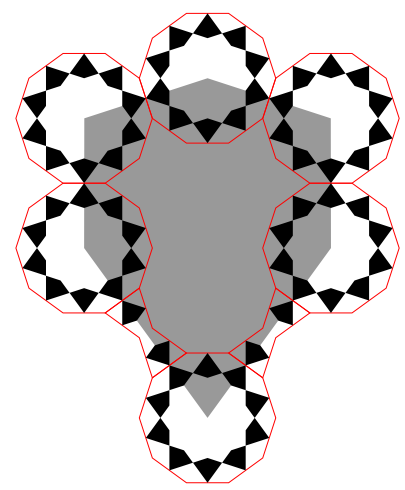

(c)

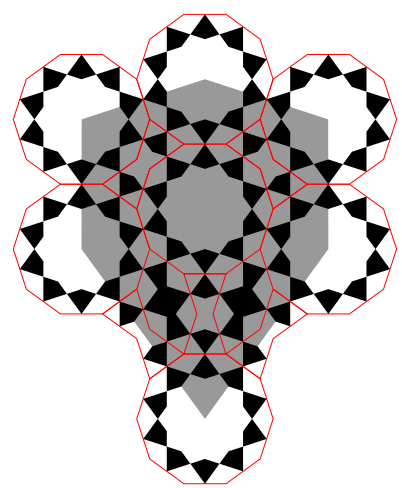

(d)

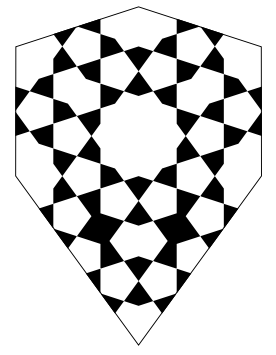

(e)

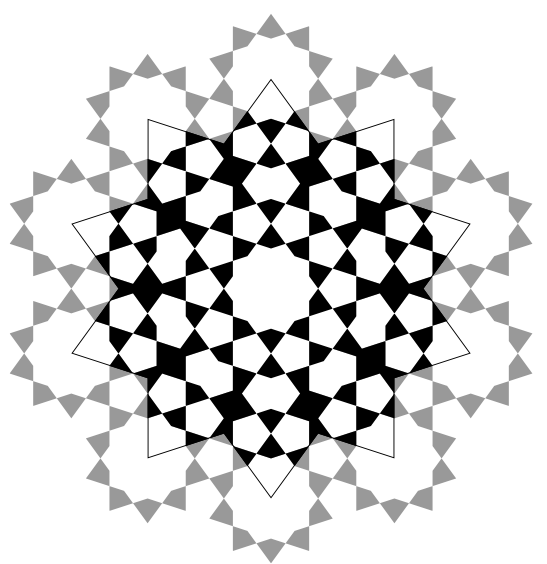

(f)

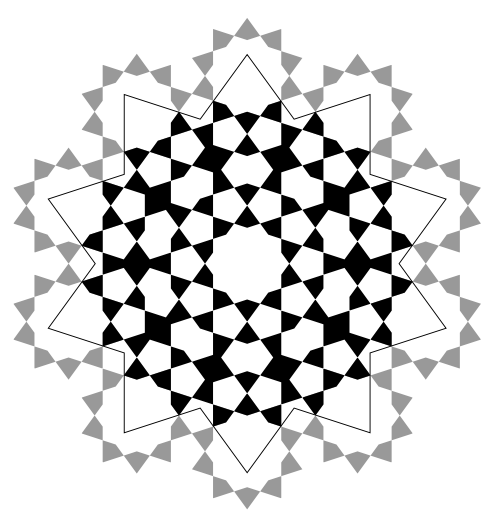

(g)

Fig. 16 Filling compartments in a spandrel design 
using a spandrel design. The large-scale pattern is a small piece of the common and easily recognised design of Fig. $1 \mathrm{~g}$. The grey triangular region highlighted in Fig. 16a is the portion used to cover the spandrel. Notice that its corners coincide with the centres of stars, and its edges are mirror lines or edges of the $\widetilde{M}\{10 / 4\}$ modules composing the pattern. These are the same rules we applied to the modular filling of the templates and we shall use them again below to fill the compartments. In (b) the spandrel region has been extracted. We shall focus on filling its white regions. As the white convex hexagons are all congruent, there are only two shapes to be considered-the hexagons and the 10-pointed stars.

Figure 16c-e shows the steps in producing a small-scale pattern to fit the hexagonal 'petal' region. In this case we use the standard triad of modules from $\widetilde{M}\{10 / 3\}$, placing a star at each corner, then completing the boundary, and finally filling the interior.

This pattern is used in a spandrel (\#32) in the Seyyed Mosque in Isfahan. The regions coloured grey in Fig. 16b-the kites, arrowheads and 5-pointed stars-are decorated with floral arabesques and the small-scale geometric pattern is applied only to the white regions. Figure $16 \mathrm{f}$ shows the small-scale pattern that is applied to the 10-pointed star. Small-scale stars have been placed at the outer corners of the compartment, but the re-entrant corners of the compartment lie inside bobbin motifs, not stars. This causes the bobbin motifs to lose their distinctive shape as they have a piece cut away from one end. To be able to place stars on all the corners of the large-scale pattern, the change in scale between the large- and small-scale patterns would need to be much larger, leading to much finer detail in the smallscale pattern. Another solution is presented in the variant of the pattern shown in Fig. 16g. It comes from the Chahar Bagh Madrasa, Isfahan, in a spandrel (\#20) above an archway that leads off the main courtyard. There the small-scale pattern is identical to that in Fig. 16f, but it has been reduced to about $90 \%$ of the size. Although this breaks the rules about the relationship between the small-scale pattern and the boundary of the compartment, the result is more satisfying as all the (black) motifs in the small-scale pattern are whole and therefore easily recognisable.

Usually, the small-scale pattern fills all the compartments of the large-scale pattern, not just alternate ones as in this example. Applying the rules about module placement (location of stars, edges and mirror lines) ensures that the small-scale pattern continues seamlessly across the whole design. If the large-scale structure is not made visible, the small-scale pattern still provides a perfectly good, if rather busy, geometric design to cover the panel.

\section{Modular Filling of Modules}

Although the undecorated modular system $M\{8 / 2\}$ appears in some very early 2-level patterns with outlined compartments (\#6, \#35), it was not applied to fill compartments until much later. It forms the basis of some field patterns with hierarchical structures dating from the Mughal period in India (Cromwell 2012a), but it was not used to fill the compartments of 2-level star patterns until the nineteenth century. Two examples from the Seyyed Mosque in Isfahan are shown in 


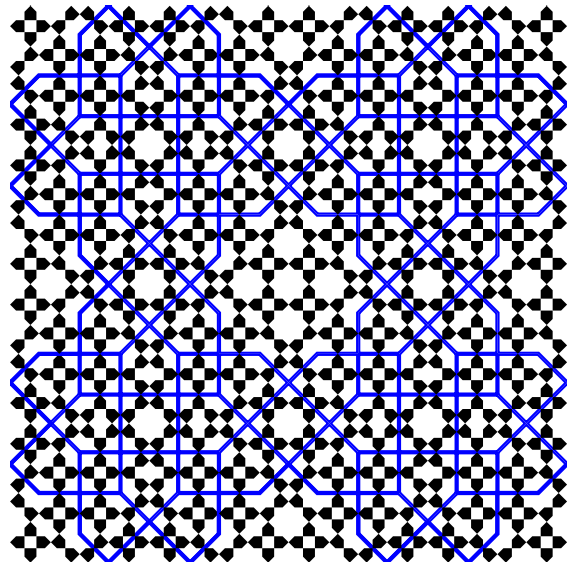

(a)

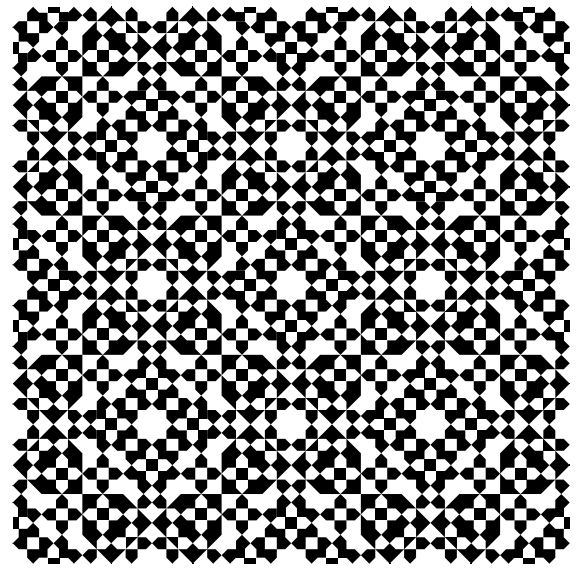

(b)

Fig. 17 Seyyed Mosque, Isfahan (1840)

Fig. 17. As these have such a late date, we might expect to find that the large-scale patterns are made visible using transparent overlay or contrast reversal. However, these styles require the large-scale pattern to have a special property: it must be possible to colour the regions in two colours so that adjacent regions have different colours-a so-called chessboard colouring. The large-scale pattern in Fig. 17a has some vertices where three regions meet so a chessboard colouring is impossiblethe artist has used the inlaid line technique for this design.

The large-scale pattern in Fig. 17b is derived from the Star and Cross pattern and is highlighted using reverse contrast. The black and white treatment used in the figure produces a rather vibrant effect. The original mosaic uses more colours to differentiate the areas: light colours (white and a sandy brown), dark colours (black and red), and a medium blue that functions as light or dark according to context. In the large house compartments, the light modules (all small houses) are brown and the dark modules are black or blue; in the other compartments, the dark modules are black and the light modules are brown or blue.

In these examples the compartments in the large-scale pattern and the large-scale modules from which it is built are the same elements. This is because $M\{8 / 2\}$ is an undecorated modular system. With the decorated modular systems, these two concepts, compartments and modules, are distinct: the compartments are either the (foreground) motifs carried by the modules or the background regions formed by fusing their corners. Hence, decorated systems provide two distinct ways to generate the small-scale pattern: fill the compartments (as in the preceding section) or fill the large-scale modules. It is possible to distinguish the two types according to whether there is consistency of layout in the compartments or in the large-scale modules. We shall show an example of each case.

Figure 18 shows the structure of the 2-level pattern produced by replicating the template in panel 32 of the Topkap1 Scroll. The large-scale pattern is composed of the pentagon, barrel and decagon modules of $\widetilde{M}\{10 / 3\}$; the modules are outlined in 


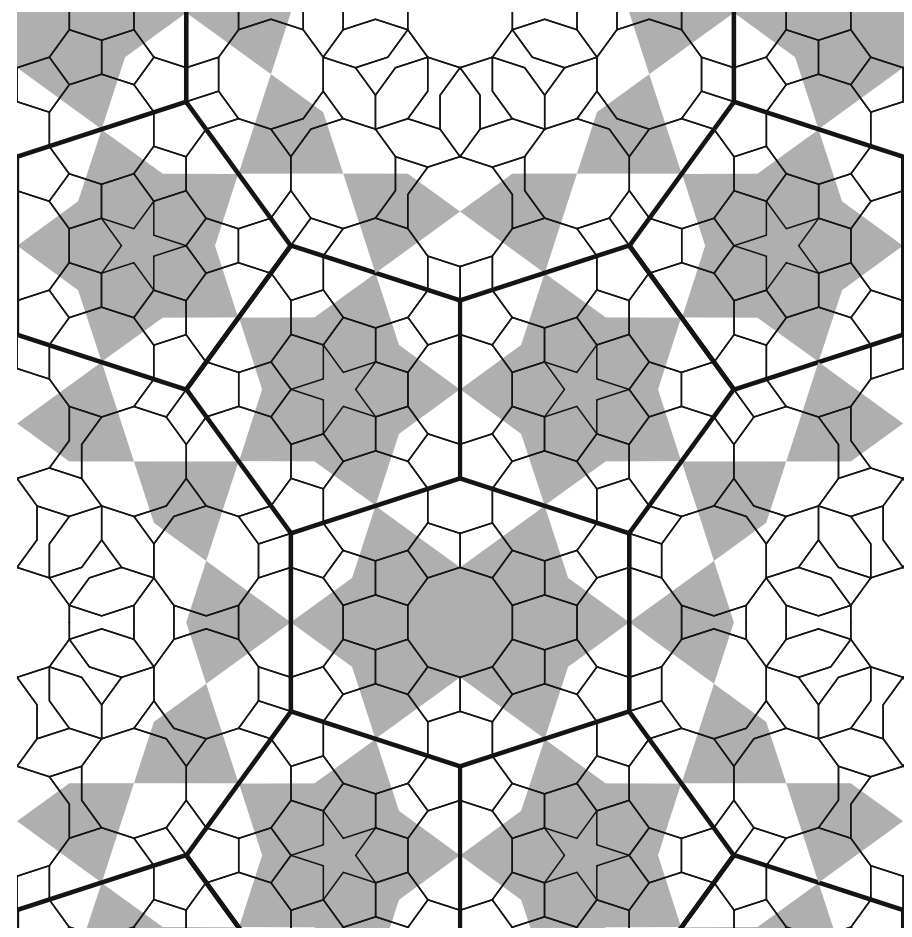

Fig. 18 Structure of panel 32 from the Topkapı Scroll

thick lines, and the motifs are shown in grey. This is the standard pattern of Fig. 1f, which is common in Central Asia but unusual in Iran. The thin lines show the modules that define the small-scale pattern. With the exception of the stars in the centre of the large-scale pentagons, the small modules also belong to $\widetilde{M}\{10 / 3\}$. The layout follows the rule we have seen before: a small-scale decagon (and hence a 10pointed star of the small-scale pattern) is centred at each crossing in the large-scale pattern, including those connecting the kites that form the outer cells of the largescale $\{10 / 3\}$ stars. The small-scale pattern (not shown in the figure) is produced by adding the standard motifs of $\widetilde{M}\{10 / 3\}$ and a non-standard adaptation to complete the middle of the large pentagons.

The white compartments of the large-scale pattern are of two shapes: 10-pointed stars of type $\{10 / 2\}$ and irregular convex hexagons. There are two different fillings of the hexagon compartments: some have decagons at three corners and contain a barrel module, others have decagons at four corners and contain rhombus modules. This lack of consistency of layout hints that an organising principle different from filling compartments may have been used but, on its own, this one example does not provide sufficient evidence to enable us to say what it is. However, this pattern is one of a set of four.

Panels 28, 31, 32 and 34 of the Topkap1 Scroll can all be constructed using the same method (Cromwell 2009, 2010c). In each case the large- and small-scale 
patterns are based on $\widetilde{M}\{10 / 3\}$ modules, and the subdivision of large modules into small ones is the same in all four patterns. Moreover, the arrangement of small modules within compartments varies both within and between patterns. The subdivisions preserve the symmetry of the large-scale modules but not the symmetry of the compartments. For example, the small-scale pattern in the pentagon compartment in panel 34 does not have 5-fold symmetry. The small-scale patterns have a consistent relationship with the large-scale modules but not with the compartments in the large-scale patterns. Because of these properties, it seems likely that these examples are based on filling the modules.

Now let us turn to an example from the Darb-i Imam in Isfahan. We shall focus on the pentagonal compartments in the large-scale pattern and explore first the different ways they could be filled. Figure 19a shows the basic framework: it has decagons containing $\{10 / 3\}$ star motifs in the corners, bow-ties along the edges, and bobbin modules radiating from the centre to the corners. Five gaps remain, each of which can be filled with one of the two mirror-image configurations shown in (b) and (c). If we regard arrangements that are related by rotation or reflection as equivalent then there are only four different ways to complete the pattern. The possibilities are shown in (d)-(g); the bow-tie modules have been shaded to highlight their distribution. In (d) the same choice is made in all five gaps, leading to a filled compartment with 5-fold rotational symmetry. In (e) four gaps have the same filling and one (the top-left) is different. Both (f) and (g) have three gaps of one kind and two of the other kind: in (f) like kinds are grouped together and in (g) they alternate when possible.

Figure $19 \mathrm{~h}$ shows the large-scale pattern from a tympanum in the Darb-i Imam; it has been overlaid with the associated large-scale modules (bow-tie and decagon from $\widetilde{M}\{10 / 3\}$ ). This is the same design as the one shown in the top-left of Fig. 2. In each pentagonal compartment the locations of the bow-tie modules corresponding to the mosaic have also been drawn in. We see that the arrangement in (g) or its mirror image is used in all the compartments.

Now let us examine the relationship of the small bow-ties to the large-scale modules. If the small-scale pattern had been produced by filling the modules, we would expect the same arrangement of small bow-ties to occur in all the large decagons, and probably a symmetrical arrangement within each decagon. However, the arrangement of small bow-tie modules in the large-scale modules is somewhat haphazard: it is not consistent between modules and does not respect the symmetry of the modules. The fact that the arrangement of the small-scale modules is consistent in the compartments but not in the large-scale modules suggests that the small-scale pattern was produced by filling the compartments rather than by filling the large-scale modules.

This has become a controversial pattern with competing claims on its method of construction. In fact, none of the proposed reconstructions is correct as they all project modern expectations of symmetry onto the design as part of the analysis and so construct a variant of the design, not a copy of the original mosaic. Bonner (2003, Fig. 16) filled the compartments with small-scale modules (different from but interchangeable with those presented here). His division of the pentagonal 


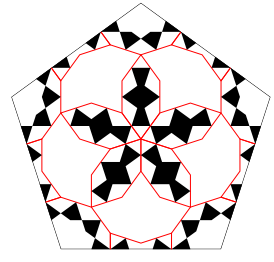

(a)

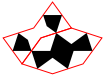

(b)

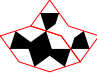

(c)

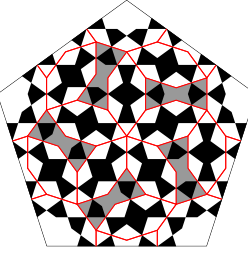

(d)

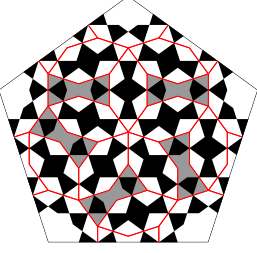

(e)

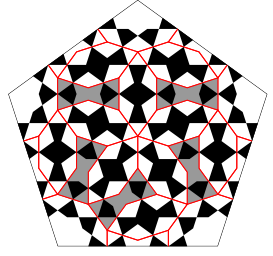

(f)

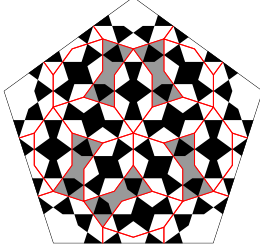

(g)

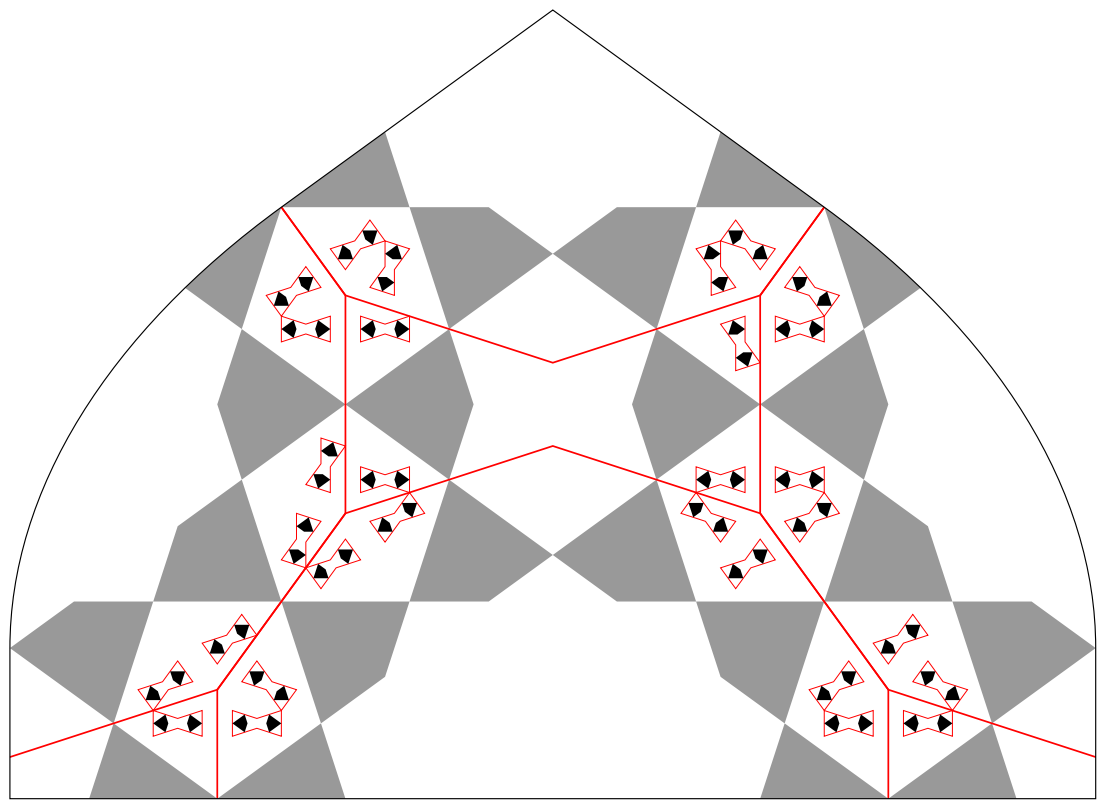

(h)

Fig. 19 Analysis of the pentagonal compartments from a tympanum in the Darb-i Imam, Isfahan

compartment has rotational symmetry and is equivalent to Fig. 19d. His figures 15-18 illustrate the compartment-filling process, but the result does not agree with the original in this minor detail. Lu and Steinhardt (2007, Fig. 3) filled the large bow-tie and decagon modules with small modules from the standard triad, and then assembled their subdivided large-scale modules in the manner of Fig. 19h to create a 2-level pattern that looks superficially like the Darb-i Imam mosaic. 
Their subdivisions have maximal symmetry and so their result does not match the original mosaic.

$\mathrm{Lu}$ and Steinhardt observed that the same set of modules is used at two different scales. As physicists, they were aware of the mathematical potential of such a relationship: overlaying the small- and large-scale module tessellations reveals how the large modules can be divided into the small ones. They assumed that this subdivision was created deliberately as part of the design process but, as we have seen, it could also be the byproduct of some other means of construction such as filling the compartments. The self-referential process of assembling shapes into larger copies of themselves is one of the mechanisms that can be used to generate patterns that have long-range structure yet do not repeat periodically. Such patterns based on small sets of shapes were discovered in the 1970s and there are now many examples. The suggestion that such patterns could have been made 500 years earlier provoked much debate. See Cromwell (2015) for a more detailed discussion of this analysis and its mathematical implications.

\section{A Non-Modular Example}

Our final example (\#11) comes from the Friday Mosque in Isfahan and is shown in Fig. 20. It is remarkable in the variety of techniques used in its construction and illustrates the difficulty of producing 2-level designs without using the modular approach.

The large-scale pattern is that of Fig. 1f - the same one as in Fig. 18. The relationship between the large-scale and small-scale patterns conforms to the standards we have come to expect: small-scale stars are centred at the crossings and

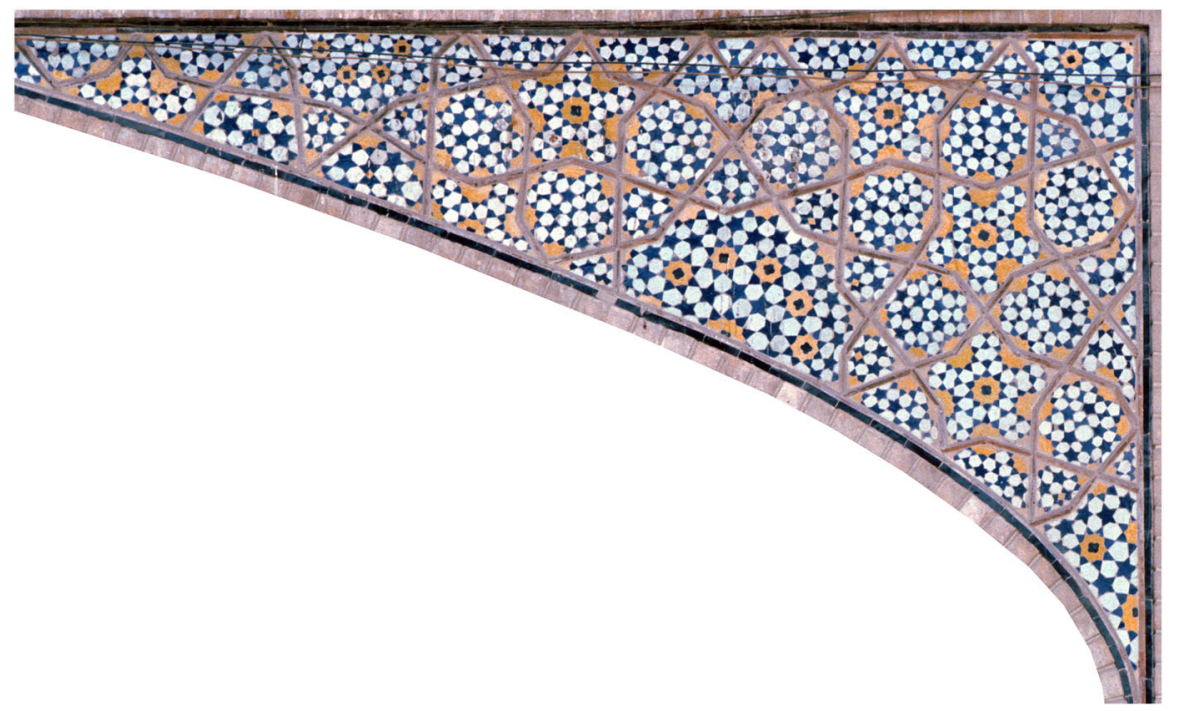

Fig. 20 Spandrel from the Friday Mosque, Isfahan. Source: photograph IRA0533 from the collection of David Wade 


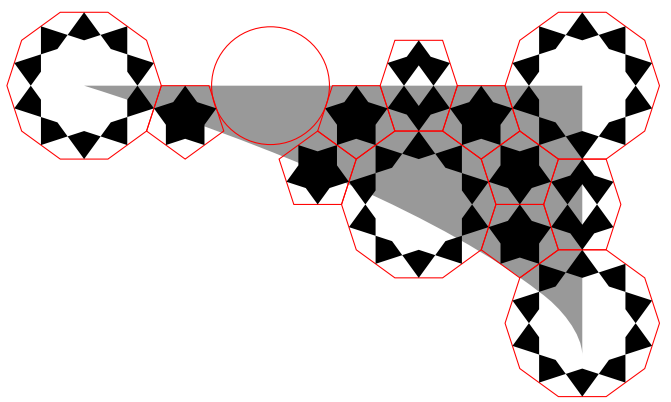

(a)

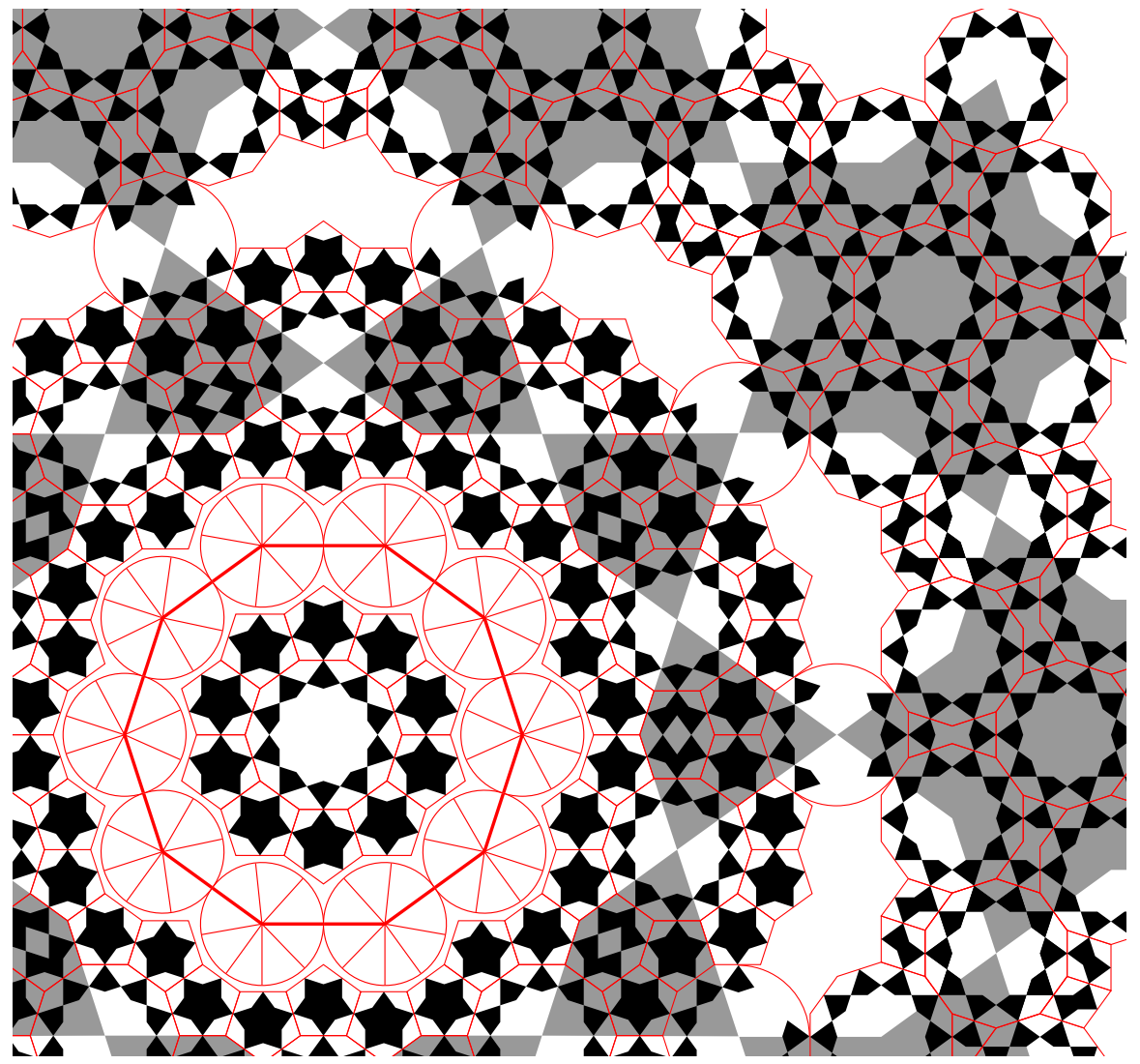

(b)

Fig. 21 Analysis of the design in Fig. 20

corners of the large-scale pattern, and the edges of the large-scale compartments are mirror lines or edges of the small-scale modules that meet them. However, the design departs from the formulaic application of the modular method in several respects.

We shall explain the deviations from the norm using the schematic representation of the design in Fig. 21. The first adjustment is applied to make the large-scale 
pattern fit the space available. The grey region in Fig. 21a shows the shape of the spandrel. The circle about one-third of the way along the top edge contains an 8-pointed star, not the 10-pointed star one expects.

Figure $21 \mathrm{~b}$ shows a detail of the design. The construction of the small-scale pattern is divided into five concentric layers centred in the lower-left corner of the figure. Layers 1,3 and 5 are covered with modules from $\widetilde{M}\{10 / 3\}$ : layer 1 consists of the central decagon module and the ten pentagons surrounding it, layer 3 covers the large-scale kites, and layer 5 covers the large-scale stars. Layers 2 and 4 are transition zones where the modular structure of the small-scale pattern breaks down.

In layer 5, along the top and right of the figure, the small-scale pattern is formed from the bow-tie and decagon modules, leading to a pattern of small black kites on the large-scale 5-pointed stars. In layer 3 this arrangement is reversed-the smallscale pattern uses the pentagon and barrel modules, leading to small 5-pointed stars on the large-scale kites.

Notice that the alignment of the small 10-pointed stars and the edges of the largescale compartments is not fixed: in layer 5 the edges of the compartments pass through the spikes of the stars whereas in layer 3 they pass between them. Layer 4 has to mediate between these inconsistent orientations; as a consequence the stars in layer 4 have eleven spikes and the other modules in this layer have been distorted and adapted to fit the space.

Layer 2 is represented in the figure by a ring of ten circles. Each circle corresponds to a star in the design. If we try to place decagon modules in these positions as a natural continuation of layer 3, we find that they overlap one another, so the 10-pointed star motifs they carry do not fit the space. In fact, each star has eight points. In the figure, the radius lines in the circles indicate the directions of the spikes of the stars. Some of the radii in neighbouring circles are aligned in pairs to form a regular decagon (shown in bold) running round the middle of layer 2. This ensures that the spikes of the stars are properly aligned. Each circle has six additional radii, two inside the decagon and four outside. Note the spacing between the radii varies - they are closest together near layer 3 and furthest apart near layer 1 . This variation induces a change of scale in the kites forming the spikes of these 8-pointed stars. As a result, the modules in layer 1 are about $5 \%$ larger than those in layer 3 .

These tricks of the trade can be found in the constructions of other Islamic patterns. The small-scale filling of the $\{10 / 3\}$ star compartments in pattern \#19 contains 8-pointed stars where ten points would be expected. The parity transition of stars from 'through spikes' to 'between spikes' (layer 4) appears in a Whirling Kites pattern (\#7) where a 13-pointed star interpolates between two orientations of 12-pointed stars (Cromwell and Beltrami 2011, Var. 5). It also occurs in pattern \#18, but here it is less obvious as only alternate compartments are filled with small-scale geometry and there is no need to interpolate between the disconnected star fragments. Irregular stars with uneven spacing of the spikes (layer 2) can be used to produce star patterns containing incompatible combinations of stars (Cromwell 2013). 


\section{Self-Similarity}

In all the 2-level examples we have analysed so far the large- and small-scale patterns are different. Even when they are both based on the same modular system, there are local configurations of motifs that appear at one scale but not in the other. I know of only one traditional Islamic pattern that uses the same pattern on two scales. This remarkable pattern, taken from a carved jali window grill in the tomb of Itimad-ad Daula, Agra, is shown in Fig. 22; photograph IND 0420 in Wade's collection (Wade 2015) shows the original. Both the large- and small-scale patterns are the periodic Star and Hexagon pattern. Notice that the structure conforms to the organising principles we have seen in the other 2-level examples-small stars centred on all the corners of the large stars, and so on. This 2-level pattern exhibits a very strong type of symmetry called scale invariance: the large- and small-scale patterns differ only in scale and orientation (enlarge by scale factor $2 \sqrt{3}$ and rotate by $\left.90^{\circ}\right)$.

Scale invariance is a very strict form of self-similarity: the different scale patterns are identical. An object is self-similar in a weaker sense if it has structure exhibiting the same general features at different scales so that when we study a detail we cannot tell which scale we are looking at. We could apply the term self-similar to patterns that employ the same modular system to construct both the large- and small-scale patterns. In these cases local configurations of modules that are used at both levels will produce the same motifs at both scales, even though globally the patterns are distinct. Examples based on the undecorated system $M\{8 / 2\}$ include patterns from Mashhad (\#35) and the Seyyed Mosque in Isfahan (\#30, \#31). In

Fig. 22 A scale-invariant design from a carved jali screen at the tomb of Itimad-ad Daula, Agra (1622-28)

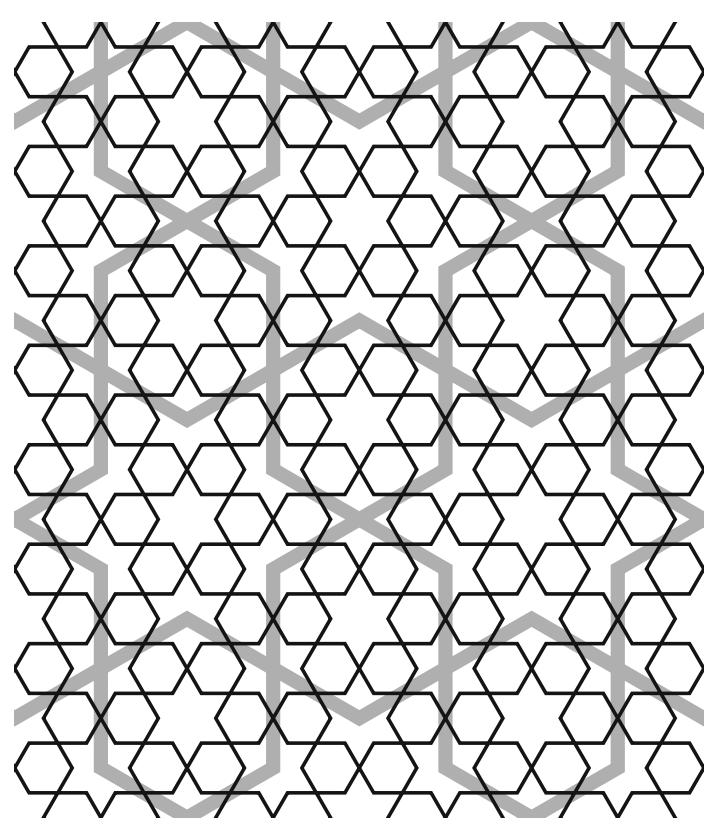


Isfahan examples based on the decorated system $\widetilde{M}\{10 / 3\}$ can be found in the Darb-i Imam (\#3, \#4, \#5), the Friday Mosque (\#10, \#11) and the Chahar Bagh Madrasa (\#16, \#17, \#18, \#19). The Topkap1 Scroll examples (\#23, \#25, \#26, \#27) are also based on this system.

Bonner (2003) also applies the term self-similar to 2-level Islamic patterns, but in a way that focuses on the structure underlying the pattern rather than the pattern itself. The two approaches can be illustrated with panel 49 of the Topkap1 Scroll (Fig. 14). In our terminology the large- and small-scale patterns are based on $\widetilde{M}\{10 / 4\}$ and $\widetilde{M}\{10 / 3\}$, respectively, which we regard as different modular systems; they are sufficiently different that we can tell them apart and hence identify which scale pattern we are looking at. Bonner regards them both as patterns constructed on a 5-fold grid and hence belonging to the same family. Whereas we consider the motifs in the pattern, Bonner looks at the shapes of the modules.

Note that 'self-similar' is not synonymous with '2-level' or 'hierarchical'. This is clear in the Whirling Kites patterns (\#9, \#8, \#7, \#22), where the techniques for filling and outlining compartments are applied to a finite geometric motif and there are no methods or motifs shared by the two levels (Cromwell and Beltrami 2011). In the examples we discussed in Sect. 4.2 the construction is based on triangular templates and the large- and small-scale patterns have no common elements. The large-scale patterns in the mosques at Balyand (Fig. 8, \#12) and Turbat-i Jam (Fig. 12, \#37) are not modular, while the small-scale patterns are applications of $M\{8 / 2\}$. A more interesting juxtaposition can be found in the Friday Mosque at Yazd (\#34), where a decorated modular system provides the small-scale pattern and the large-scale pattern is based on the undecorated $M\{8 / 2\}$. The modular system used to construct the small-scale pattern may be an adaptation of the $\widetilde{M}\{10 / 3\}$ system to the 8-fold setting-see Cromwell (2012b) for a detailed discussion.

\section{Multiscale Ornament}

We have focussed on geometric examples that started to appear in the fifteenth century, but the interplay of multiple themes at different scales was an early feature of Islamic ornament. The early methods were rather simple, often just a matter of progressively filling voids in the background with floral or vegetal scrolls to leave a design with no vacant spaces. There are examples of this type in the ninth-century al-Tariq Mosque (Masjid-i Nuh Gunbad) at Balkh, Afghanistan: geometric strapwork patterns outline spaces filled with palmette foliage; the straps are also decorated with a sequence of dots drilled along their length. The soffit of the twelfth-century arch at Qal'a-i Bust, Afghanistan, is decorated with the common star pattern of Fig. $1 \mathrm{~g}$ and the compartments are filled with floral motifs on terracotta plates. This technique is also used in the thirteenth-century Mustansiriya Madrasa in Baghdad, which has many beautiful 2-level designs of polygonal networks or star patterns outlined in thin lines, with arabesque inserts filling the compartments. 


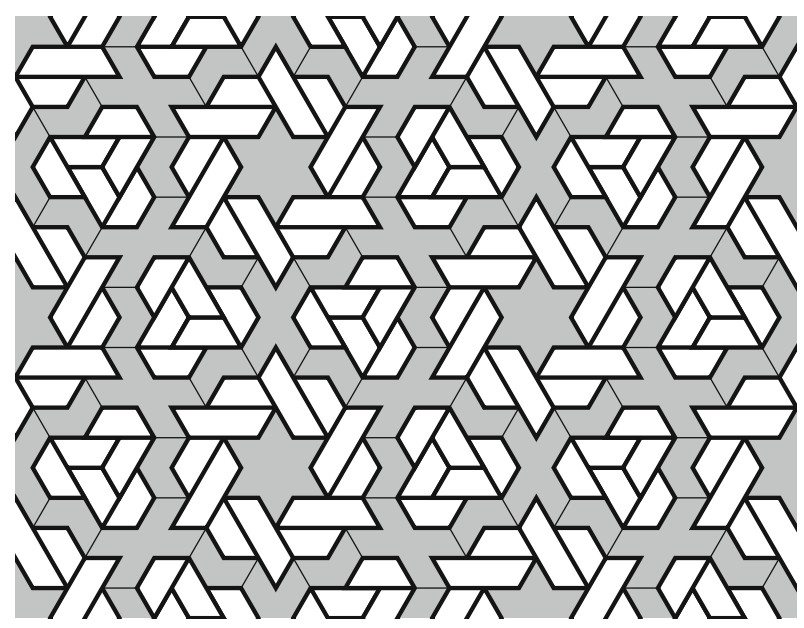

Fig. 23 Pattern from the Mustansiriya Madrasa, Baghdad

A design from the soffit of an iwan in the madrasa is shown in Fig. 23. The dark areas of the figure are covered with arabesque in deep relief and the white areas of the figure are filled with smooth trapezia arranged like bricks. The shadows cast by the relief make the large-scale pattern visible as dark bands against the smooth, reflective surfaces of the bricks. This design is unusual in several respects. First, the use of motifs with rotational symmetry but no mirror symmetry is rare in Islamic ornament (earlier we noted the avoidance of cheiral configurations like Fig. 19d). Second, rather than filling the compartments, the arabesque plays the outlining role.

The linear format of calligraphy makes it a natural candidate for use in framing compartments. The lower section of the minaret at Jam in Afghanistan (1190) is divided into eight vertical panels, each of which has geometric compartments outlined with a band carrying text. In one panel the large-scale framework has the same form as that in Fig. 1b, the adjacent panel contains 8-pointed stars like those in the Star and Cross pattern, and the next is a compass work design. In these examples the path of the calligraphy is more interesting than the routine rectangular frame. However, the small-scale geometric patterns used to fill the compartments are just cropped to fit-the large- and small-scale patterns are not yet related and their key points do not coincide.

A stylised form of the Kufic script was developed for covering large areas with ornamental calligraphy. It is organised on a square grid and is known as square or geometric Kufic. The Nimavard Madrasa in Isfahan, and the Mir Chaqmaq Mosque in Yazd (1437) both contain examples of 2-level Kufic calligraphy-a short word, usually a name, is written in a large script, and a long text, such as a sura, is written in a much smaller script along the paths defined by the large letters (http://www. kufic.info/).

The production of multiscale ornament is not unique to the Islamic tradition. In fact, ornament that contains a hierarchy of patterns of different scales is found in many cultures and is known as hypotactic ornament (Trilling 2001) — a term derived 
from the Greek roots 'tassein' (to arrange) and 'hypo-' (beneath). Some of these designs are possible precursors and influences on the Islamic examples. For example, describing the geometric elements of classical mosaics, Dunbabin writes (Dunbabin 1999, p. 291).

The designs range from very simple arrangements of basic geometric forms to immensely complicated combinations where one pattern is superimposed upon another and subsidiary motifs are used to outline or to fill the basic elements.

The complexity of classical ornament is low when compared with Islamic designs, but the same principles for organising a composition, filling and outlining, are apparent. In this respect, Islamic 2-level ornament is a continuation and development of classical Roman and Byzantine examples.

Figure 24a shows the geometric elements from a Roman mosaic in SaintRomain-en-Gal, Vienna. The large-scale pattern is a framework of regular hexagons; the pathways outlining the compartments are covered with a simple arrangement of hexagons, and 6-pointed stars divided into rhombi. This network frames the primary motifs: the compartments are edged in a 3-string braid and display figurative designs of flowers-see Dunbabin (1999, Fig. 78) for a photograph of the original. The catalogue of patterns from Roman mosaics (Balmelle and Prudhomme 1985) has other examples in which Archimedean tilings are used to provide the compartmental framework: these include vertex types 3.4.6.4 (Fig. 205), 3.6.3.6 (Fig. 209) and 4.8.8 (Fig. 164).

In these classical applications of the outlining method, the geometry always plays a strictly supporting role-it is just used to organise the space. In some Islamic patterns the division into foreground and background, into primary and secondary roles, is more ambiguous and neither element dominates. Attention can switch from

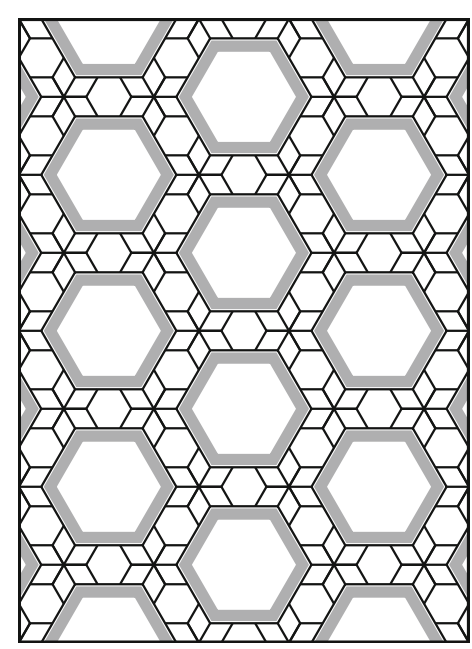

(a)

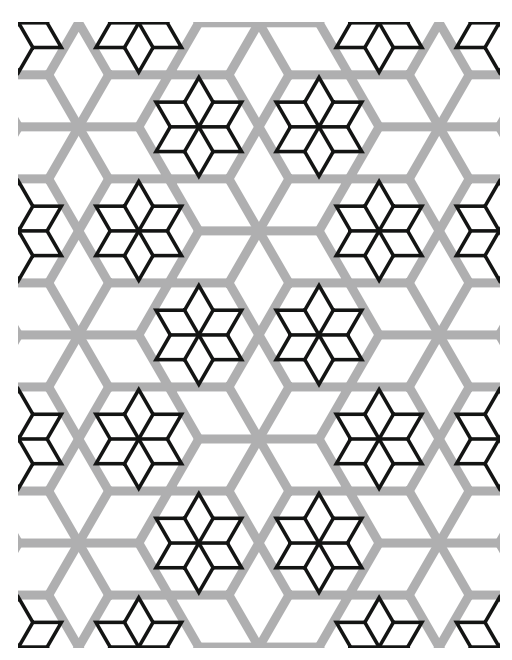

(b)

Fig. 24 Other examples of multiscale ornament 
one to the other without conflict or tension. This complementary nature of two elements is also achieved in the best examples of 2-level geometric design.

The Cosmati pavements in thirteenth-century Italy are another development of the Byzantine tradition. Cosmati work is characterised by a large-scale framework in white marble, often a simple compass work design in the form of a strip of guilloche or a composition of tangent circles in a square panel like a quincunx, with the compartments filled with intricate geometric patterns made of pieces cut from coloured stones and glass. Another common feature of the patterns is the recursive subdivision of any triangular regions into smaller triangles in the manner now associated with the fractal Sierpinski gasket. This subdivision process creates regions with a range of sizes, allowing offcuts and small fragments of the scarce coloured marbles to be employed. In some designs there is evidence of Islamic influence in the choice of the large-scale pattern. For example, the twelfth-century floor of the Baptistery in Pisa uses a classic Islamic pattern of interlaced trefoil knots (Pajares-Ayuela 2001, Fig. 1-26). More examples can be found in and around Palermo, Sicily, in the church of San Cataldo, the Palatine Chapel, and Monreale Cathedral. Although not a Cosmatesque design, Fig. 24b shows another 2-level pattern from the same region-a window grill from the Zisa Palace.

A Persian form of marquetry known as khatam-kari is of particular interest here because of its construction process. The intricate patterns are constructed from thin strips or beads of wood, bone, ivory or metal. The beads are grouped into bundles and glued to form rods, the rods are assembled into larger bundles, and so on. The bundles are sometimes wrapped in metal foil, which introduces larger-scale structures similar to compartments into the design. The desired pattern appears as the cross-section of the resulting block. Thin slices of the block are then applied to boxes and other small objects for decoration. A typical pattern will have hundreds of pieces per square inch. Wulff (1966, pp. 92-97) describes the procedure in more detail.

The khatam-kari patterns are based on a modular system of three shapes (see Fig. 25a): a rhombus with angles $60^{\circ}$ and $120^{\circ}$, and the two triangles that are produced by bisecting the rhombus along its diagonals - an equilateral triangle, and a $30^{\circ}-120^{\circ}-30^{\circ}$ isosceles triangle. These basic shapes are grouped together to make larger copies of themselves ((b) and (c)) and a regular hexagon (d). The shapes in this extended set can be assembled to make 6-pointed stars of type $\{6 / 2\}$ ((e) and (f)), which can then be padded out to form larger hexagons ((g) and (h)). The process can be iterated with the composite shapes (the bundles) to generate larger copies of the same simple forms but with increasing internal complexity. The penultimate step of the process must produce rods with triangular or hexagonal cross-sections, which are then packed in the configuration shown in Fig. 25h to form the final block. This process is clearly modular and hierarchical.

The khatam-kari technique is at least 700 years old and may be derived from Byzantine prototypes. Scaled up versions of patterns in the same family can be found in Cosmati work. A large panel of this type decorates a tomb in the thirteenth century cloisters of San Giovanni in Laterano, Rome (Pajares-Ayuela 2001, Fig. 2240). Further examples from the church of San Lorenzo Fuori le Mura, Rome, are 


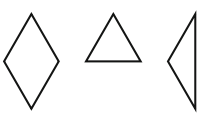

(a)

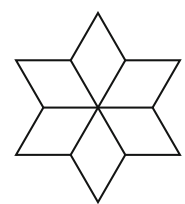

(e)

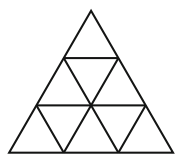

(b)

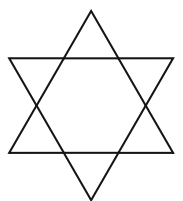

(f)

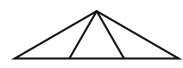

(c)

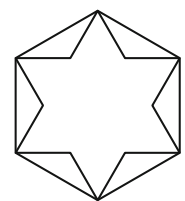

(g)

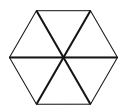

(d)

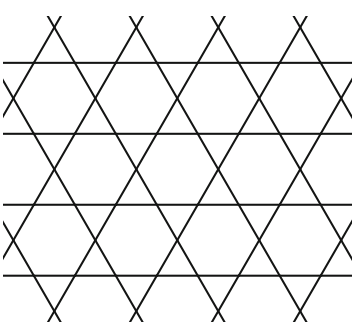

(i)

Fig. 25 Modules and hierarchies in khatam-kari pattern construction

included among the patterns in Plate 30 of Jones' Grammar of Ornament, where they are listed as Byzantine.

All of these examples show that the geometric 2-level patterns we have analysed are part of a much wider exploration of 2-level ornament.

\section{Conclusions}

We have demonstrated a variety of methods that can be used to create traditional Islamic geometric ornament in which complementary patterns of different scales are combined in one design. In the cases we have examined here a small-scale pattern is used to fill or outline the compartments in a large-scale pattern. There are also a few examples (\#16, \#33, \#34, \#38) in which the small-scale pattern runs continuously over a whole panel and serves both roles: it covers the pathways that outline the compartments and extends seamlessly to fill the compartments; the filling and outlining functions are distinguished only through contrast reversal and colour. A fine example of this type decorates the tympanum over the main entrance to the Friday Mosque in Yazd (\#34).

The methods have been presented in an order that is suggestive of a progressive logical development: there is a general increase in complexity of both the techniques and the resulting patterns. The order correlates fairly well with the dates 
attributed to the original patterns, although some anomalies are discussed below. However, sorting the mosaics by date is difficult because patterns are replaced as fashions change, and buildings are redecorated or restored. Even when we can ascribe dates with some certainty, there is still the problem of establishing whether similar methods are independent discoveries and, if not, tracing the influence and relationships between them. We have not treated these art historical questions in the text but we shall speculate here.

There is an intrinsic difference in complexity between filling and outlining. Filling is a one-step process: once the large-scale pattern has been chosen, the compartmental framework is fixed - the problem is to fill the compartments with a small-scale pattern. Outlining (using the methods of Sects. 4.2, 4.3) is a two-step process in which the templates that cover the pathways are an additional layer of structure that mediates between the large- and small-scale patterns. The templates pave the pathways that form the large-scale pattern and they must be filled with a small-scale pattern. These two steps are independent and can be performed in either order:

(a) start with a set of templates that are filled with a small-scale pattern and assemble them to make pathways

(b) subdivide the pathways of the large-scale pattern to create a set of templates, then find small-scale fillings of the templates.

Both of these outlining methods have been used. The early patterns seem to use (a) and the later ones use (b).

It is not necessary to regard outlining as a two-step process. Indeed, the earliest technique is based on strip patterns and mitred corners (\#35). However, the mitring introduces spurious shapes into the small-scale pattern; the use of intermediate templates overcomes this undesirable side-effect. For the patterns we discussed in Sect. 4.2 the small-scale filling and the template come ready-made from an existing pattern constructed on a triangular grid. In Sect. 4.3 we used small sets of templates with simple fillings; the large-scale pattern is assembled from available templates, and this limits the choice of large-scale pattern, often resulting in an unfamiliar pattern and pathways that are off-centre from the skeleton.

By the time of the Topkap1 Scroll, the templates are fitted to a given large-scale pattern. This requires a larger number of templates: the construction of the extract from panel 49 (Fig. 14) used three templates; Bonner used five for the full panel (Bonner 2003). This method allows the large-scale pattern to be selected at the start, so it can be familiar and easily recognisable. The pathways can also be centred on the skeleton. Furthermore, the method extends easily to produce the 2-level patterns with combined outlining and filling modes: the pathways and compartments in the tympanum at Yazd can both be assembled from the same set of four templates (Cromwell 2012b). Modular systems facilitate the production of small-scale fillings of the required templates. Applications of modular filling can become rather formulaic and the Chahar Bagh Madrasa, Isfahan, has many examples of 2-level patterns produced in this way (not all are listed in the catalogue). Using the Whirling Kites motif as the large-scale pattern provides more of a challenge as the large- and 
small-scale geometries are not naturally compatible and some ingenuity is required to reconcile them (Cromwell and Beltrami 2011).

\section{Small-Scale Star Placement}

Before Islamic artists started producing 2-level geometric patterns, they had a well established formula for filling rectangular panels with star patterns: place a star in each corner and fill the interior with a symmetrical arrangement of other shapes, usually with a star or other prominent feature in the centre. When a modular approach was used, the edges of the panel were covered with the edges or mirror lines of modules. This meant that if the panel were repeated as a template by reflection in the sides of the rectangle, the pattern would continue seamlessly across the joins. The leap to 2-level patterns was accomplished by applying this formula not to simple rectangles, but to the variously shaped compartments in existing Islamic geometric patterns. In general, this is difficult to do, but modularity provides a simple mechanism by which it can be achieved.

In the introduction we listed the general principles that govern the relationship between the large- and small-scale patterns. One of the most widely followed rules is that small-scale stars are centred on key points (crossings and corners) in the large-scale pattern. The exceptions generally arise because of incompatibilities in the underlying geometry. For example, some key points in the large-scale pattern may be too close together to allow small-scale stars to fit without overlapping. This happens in the $\{5 / 2\}$ and $\{10 / 4\}$ stars in Fig. $16 b$ - the change in scale between the large- and small-scale patterns is too small. To accommodate this the ratio must be at least $6+2 \sqrt{5} \approx 10.472$, as in pattern $\# 21$.

Another problem arises in some of the Whirling Kites patterns with outlined compartments: a choice must be made between having stars centred on all the corners of the pathways but with irregular spacing of stars along the edges of the pathways (as in \#7), or having stars evenly spaced along the edges of the pathways as far as possible but missing some of the corners (\#8). The pathways produced by mitring strip patterns (\#12, \#35) are more examples where some of the corners of the pathways do not coincide with star centres.

\section{The Yazd Pattern}

The 2-level design in the tympanum of the portal at the Friday Mosque in Yazd is an imaginative and harmonious fusion of contrasting modular methods (Cromwell $2012 b$ ). The large-scale pattern is constructed from the (undecorated) $M\{8 / 2\}$ system; the design is related to the common pattern used on either side of the mihrab, but has been adapted to fit the shape of the tympanum. The small-scale pattern is constructed with a decorated modular system that could be labelled $\widetilde{M}\{8 / 3\}$ with tiles and motifs modelled on the $\widetilde{M}\{10 / 3\}$ system and modified to accommodate the 8 -fold setting. The edges of the tiles are of two lengths, but the ratio is not a common one like $\sqrt{2}$ or the golden ratio we have seen in the other systems. In fact, the edge lengths are very close: the ratio is 
$\cos \left(22.5^{\circ}\right)=1 / 2 \sqrt{2+\sqrt{2}} \approx 0.924$. The small-scale pattern covers the whole panel and performs both outlining and filling of the large-scale pattern, using contrast reversal to differentiate the two functions.

The strict and austere straight line geometry of the tympanum pattern contrasts strongly with the fluid and playful decoration that covers the rest of the portal. Most of the tilework in the mosque dates from the end of the fourteenth century and is Timurid in style. However, the entrance was repaired and restored in 1457 (Blair and Bloom 1995, pp. 50-54) and the tympanum may have been redesigned at this time.

In the fifteen century, Iran was ruled by rival Turkmen tribes: the Qara Qoyunlu (Black Sheep) and the Aq Qoyunlu (White Sheep). Their territory originally covered Azerbaijan, Armenia, eastern Turkey, Iraq and north-west Iran but grew eastwards into central Iran, taking Yazd from the Timurids in 1452. The early 2-level patterns in Isfahan were created under their patronage: the Darb-i Imam was built by Jahan Shah (Black Sheep) in 1453 (\#3, \#4, \#5) and the tilework of the south iwan of the Friday Mosque was repaired by his successor, Uzun Hassan (White Sheep), in 1475 (\#6, \#7).

The Blue Mosque in Tabriz has another 2-level pattern from this period. Tabriz in north-west Iran was the capital of the Black Sheep tribe, then the White Sheep, and also the early Safavids. The Blue Mosque was built in 1465 on the order of Jahan Shah, but collapsed in 1779 when the region suffered a severe earthquake. It has been rebuilt from the ruins and fragments of the original high quality tilework remain. Like at Yazd, much of the decoration is Timurid in style (floral arabesques and calligraphy) but, high on the right-hand outer side of the entrance, is a square panel of geometric design (\#40). It is a Whirling Kites pattern with $45^{\circ}$ kite angle. Only half remains but it is possible to reconstruct the whole design-see Fig. 26. The small-scale pattern can be constructed using the same $\widetilde{M}\{8 / 3\}$ modular system as the Yazd tympanum.

\section{The Topkapı Scroll}

Tilework is not our only source of examples of 2-level patterns. The Topkap1 Scroll includes rectangular templates for generating geometric patterns, seven of which produce 2-level designs: two with outlined compartments and five with filled compartments. None of these 2-level patterns is known from any other sources. In fact, they display a high degree of ingenuity and imagination, and are unconventional in some respects. Their constructions extend the methods we have seen in our analyses of surviving mosaics and they may be the result of someone exploring possibilities rather than a record of best practice.

In Sect. 4.2 we described a method that takes any pattern constructed on the standard triangular grid and produces a 2-level pattern by omitting some of the repeat units. Panel 38 of the scroll follows a similar principle, but is built from two related patterns, one constructed on a triangular grid, the other on a square grid. The patterns in the triangular and square repeat units are compatible along the edges; they are assembled as shown in Fig. 13b to form the pathways in panel 38. (The 


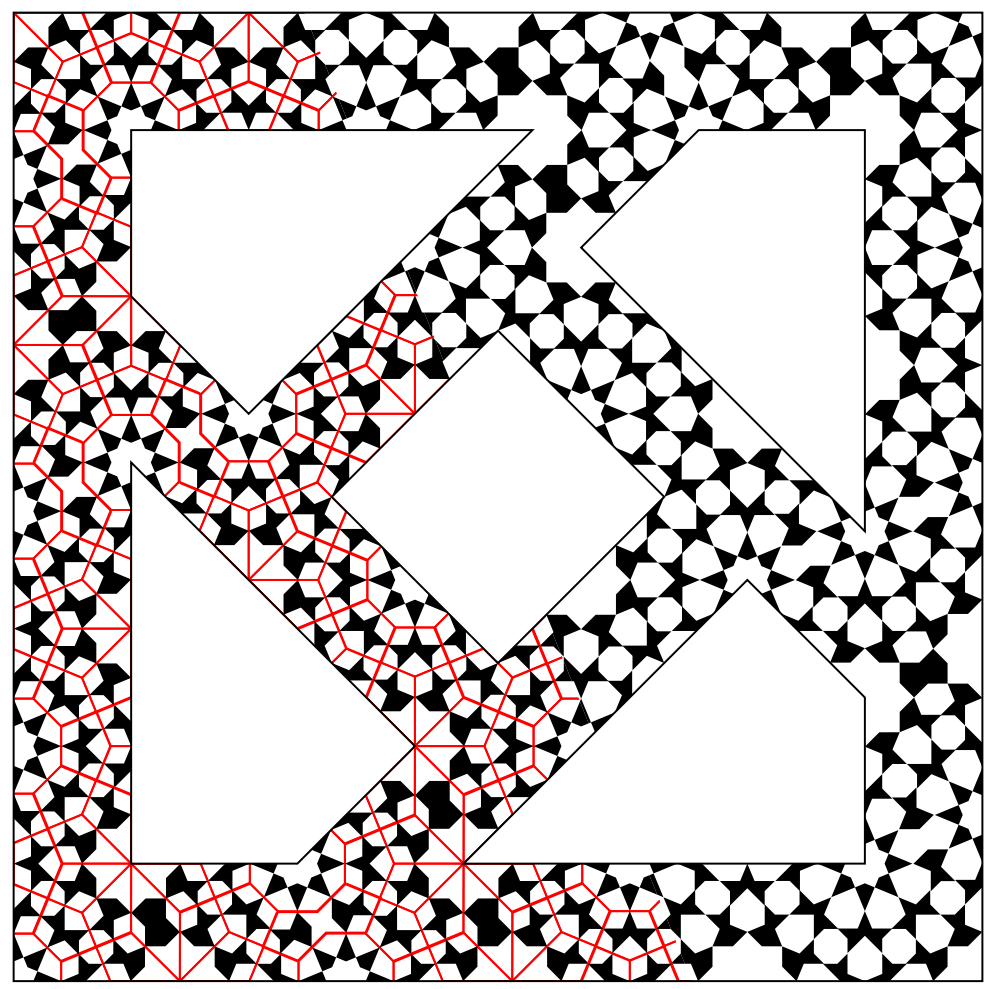

Fig. 26 Whirling Kites pattern from the Blue Mosque, Tabriz (1465)

same two templates are combined in a 1-level design in panel 35 of the scroll Cromwell 2010b.)

The other example with outlined compartments is from panel 49-see Fig. 14. As we noted in Sect. 4.3, the large-scale pattern is a more complex star pattern than is found in the mosaic examples, yet the small-scale pattern is so well-behaved that the skeleton of the large-scale pattern follows the centre-line of the pathways. This degree of complexity and control is not found in the mosaics until the outlining and filling modes are combined in the 'continuous carpet' form of 2-level patterns (for example \#16, \#33 and \#34).

Panel 29 is an example of filled compartments. The large-scale pattern is organised on the standard decagon and bow-tie grid that underlies Fig. 2. In this case, the bow-ties carry the motif from the bow-tie module in $\widetilde{M}\{10 / 4\}$ (Fig. 5) and the decagons are decorated with stars of type $\{10 / 2\}$. The small-scale pattern is constructed from $\widetilde{M}\{10 / 3\}$ : small-scale stars lie on the crossings and most of the corners in the large-scale pattern, and the design is completed by filling the compartments (Cromwell 2010c, Fig. 18). Because the $\{10 / 2\}$ stars have blunt spikes, there are large voids in the star interiors where the small-scale pattern is not interrupted by features of the large-scale pattern. The other compartments are small 
in comparison. This uneven distribution of compartment sizes means the result is not visually satisfying.

The other four panels with filled compartments-28, 30, 32 and 34-form a family of patterns with the same underlying method of construction. They seem to have been created by filling the large-scale modules rather than filling the compartments in the large-scale pattern. Both the large- and small-scale patterns are based on $\widetilde{M}\{10 / 3\}$. The same subdivision of large modules into small ones is used in all four patterns. The large rhombus, barrel, bobbin and decagon modules are filled without gaps, but the pentagon and bow-tie cannot be subdivided without introducing extra shapes. The subdivisions of the large modules do contain small bow-ties and pentagons, so this subdivision scheme cannot be used recursively to create quasiperiodicity (Cromwell 2009). None of the patterns in the catalogue is quasiperiodic.

Besides their novel method of construction, these panels are also unusual in the choice of large-scale pattern. Only panel 32 (Fig. 18) uses a familiar pattern (Fig. 1f). The other three patterns all include the large rhombus module and are not known from other sources. Panel 34 does not contain the large decagon module, so the large-scale pattern is a field pattern (has no stars).

In her study of the scroll, Necipoğlu (1995) writes that it was probably compiled in the late fifteenth or sixteenth century, somewhere in western or central Iran, under Turkmen or early Safavid patronage. All of these conclusions are consistent with the analysis presented here.

\section{Self-Reference}

In this paper we have seen that grouping modules together to form larger ones introduces hierarchical structure into a composition. Hierarchy can also be found in the geometric ornament of other cultures. For example, the abstract decoration of Celtic manuscripts exhibits the same horror vacui, the progressive filling of background shapes with ever finer detail. As in Islamic ornament, the different canons of ornament (spirals, key patterns, knotwork, zoomorphs) are usually segregated and not mixed within a compartment. In the Celtic examples a reduction in scale is often associated with an increase in complexity: the large-scale elements use simpler compositional elements (rectangles, crosses, initials) than the smallscale filling in the compartments. The Islamic examples of 2-level design are distinguished by the use of complex patterns at both scales, and the close geometric relationship between the large- and small-scale patterns.

Islamic patterns contain not only hierarchy, but also self-reference. This occurs when the same modular system is used to produce the large- and small-scale patterns. The same local configurations and motifs will appear at both scales, although they may be organised in different ways at the two levels. This is what we called self-similarity in Islamic patterns.

There are instances of self-similarity in other geometric art. For example, guilloche ribbons decorated with guilloche on a smaller scale-sequences of small twists running along twisted paths. Self-similar patterns usually involve a recursive 
process that generates finer detail of the same form. The Sierpinski triangle design found in Cosmati pavements is a good example of medieval recursion-a mosaic in San Gregorio Magno, Rome has three iterations (Pajares-Ayuela 2001, Fig. 4-114). An eleventh-century Celtic key pattern carved in a stone cross in Wales also displays recursive properties (Gailiunas 2011). However, these direct applications of self-reference are isolated. The Islamic practice is more subtle-it is a stylistic reference. Furthermore, it is only in Islamic ornament that a systematic method was applied to create a wide variety of hierarchical designs, and a modular approach seems to have been the key.

\section{Catalogue}

The catalogue lists data about the patterns mentioned in the text. It is not an exhaustive list of 2-level patterns, but it contains all of the early examples that I am aware of. There are further examples in the Chahar Bagh Madrasa and the Seyyed Mosque in Isfahan, the shrine of Fatima Masumeh in Qom, and the Vakil Mosque in Shiraz.

In the catalogue the patterns are grouped by location since sorting them by date is problematic. The columns contain the following data:

1. The index number that we have used throughout the text.

2. Description of the location of the pattern.

3. References to figures or photographs of the pattern in this paper or elsewhere.

4. Method of construction. Following Bonner (2003) we designate filling and outlining as $\mathrm{A}$ and $\mathrm{B}$, respectively. We use $\mathrm{A}+\mathrm{B}$ for the patterns that combine both modes. These types are subdivided as follows.

- $\mathrm{Ai}=$ filling, inlaid line

- Ao = filling, transparent overlay

- $\mathrm{Ar}=$ filling, contrast reversal

- $\mathrm{Ax}=$ filling, alternate regions only (one colour of a chessboard shading)

- $\mathrm{Bm}=$ outlining, mitred corners

- $\mathrm{B} \triangle=$ outlining, templates from a triangle-based pattern

- Bo = outlining, templates, paths off-centre from skeleton

- $\mathrm{Bc}=$ outlining, templates, paths centred on skeleton.

5. The large-scale pattern if it is a common one.

6. The modular system for the large-scale pattern.

7. The modular system for the small-scale pattern. The standard triad of modules from $\widetilde{M}\{10 / 3\}$ is so common that its use is listed separately.

8. The scale-factor between the large- and small-scale patterns when this is welldefined. This usually means the two modular systems are the same, but it can also be calculated between $\widetilde{M}\{10 / 3\}$ and $\widetilde{M}\{10 / 4\}$ as both systems contain a regular decagon.

9. References to other literature where the pattern is discussed (Table 1). 


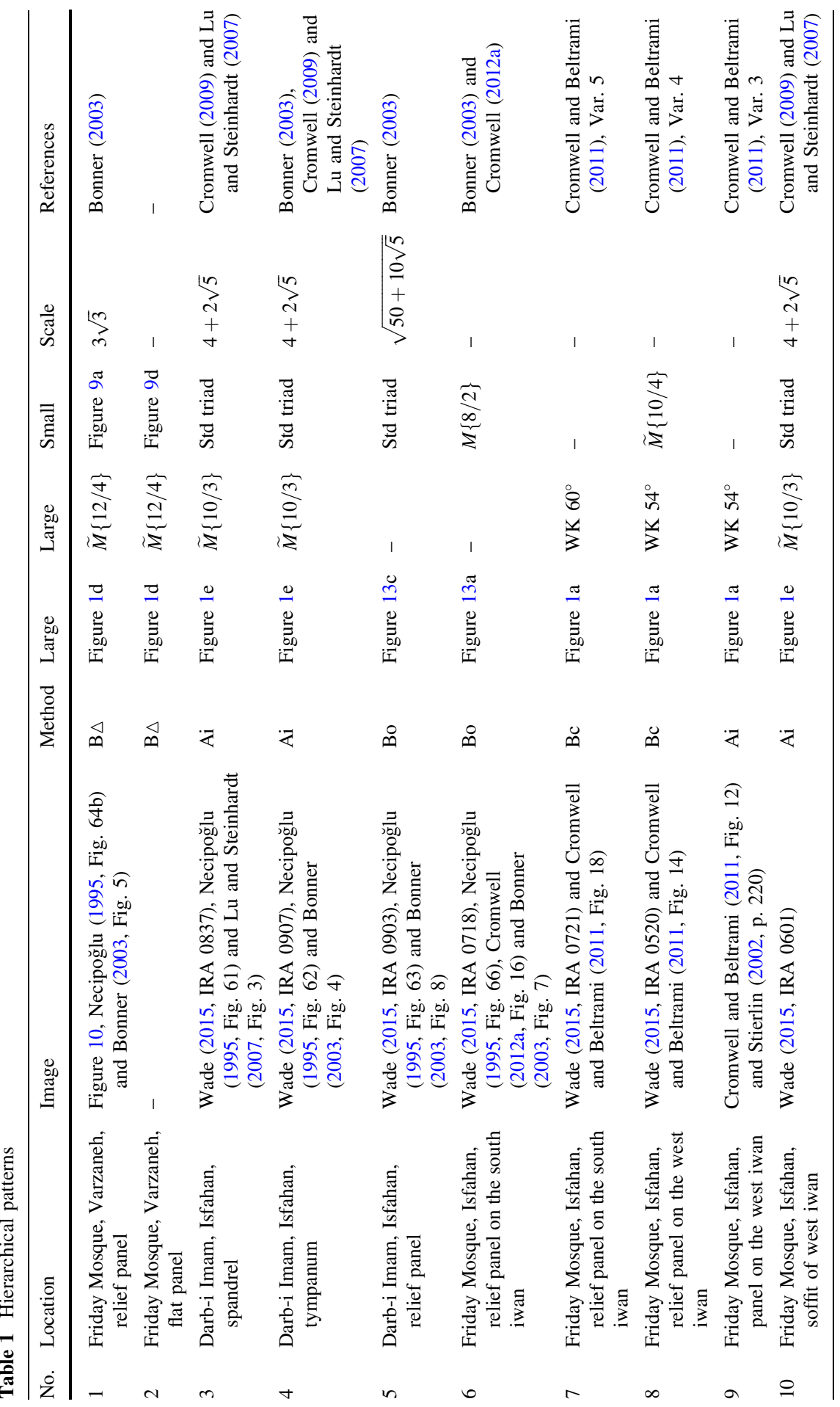




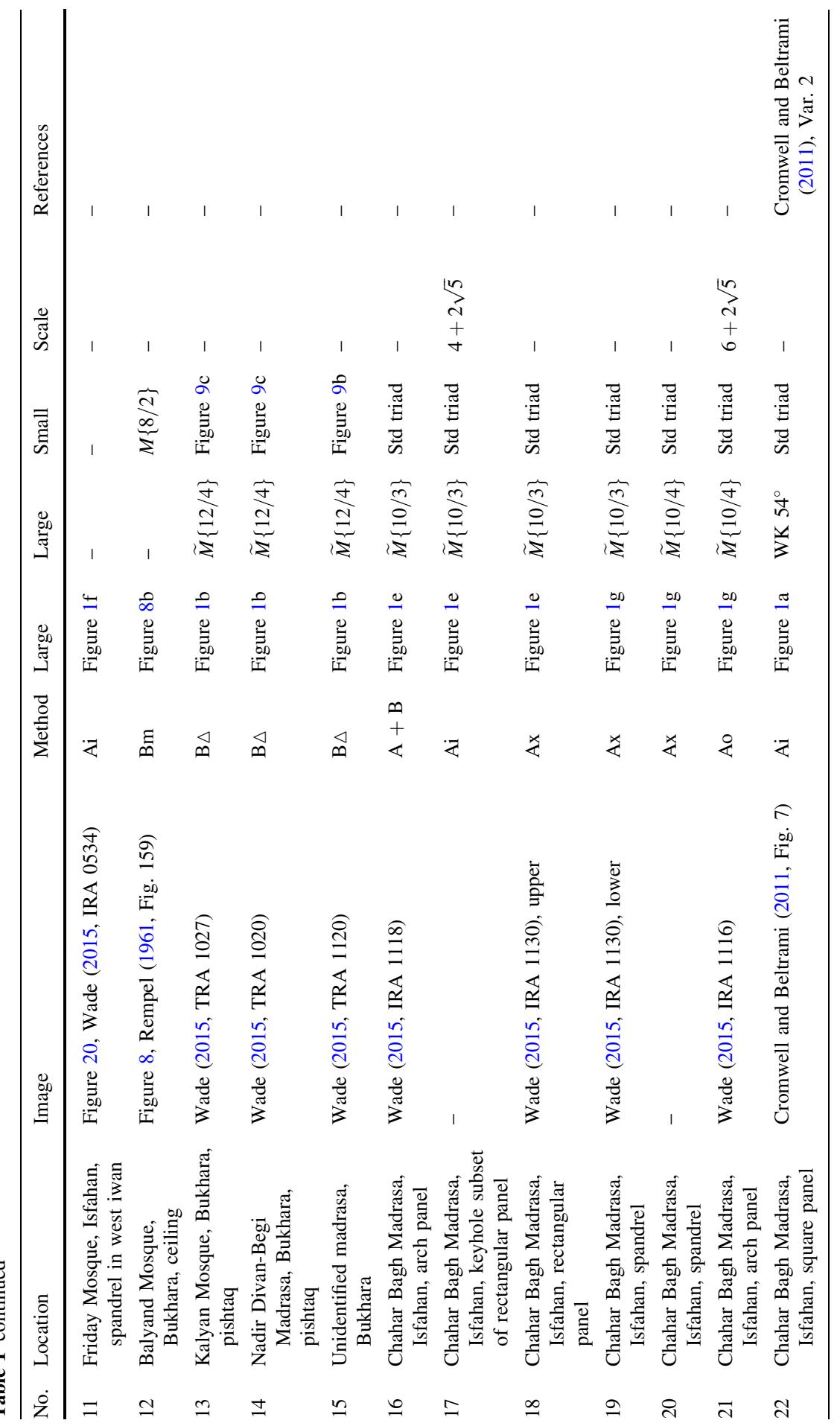




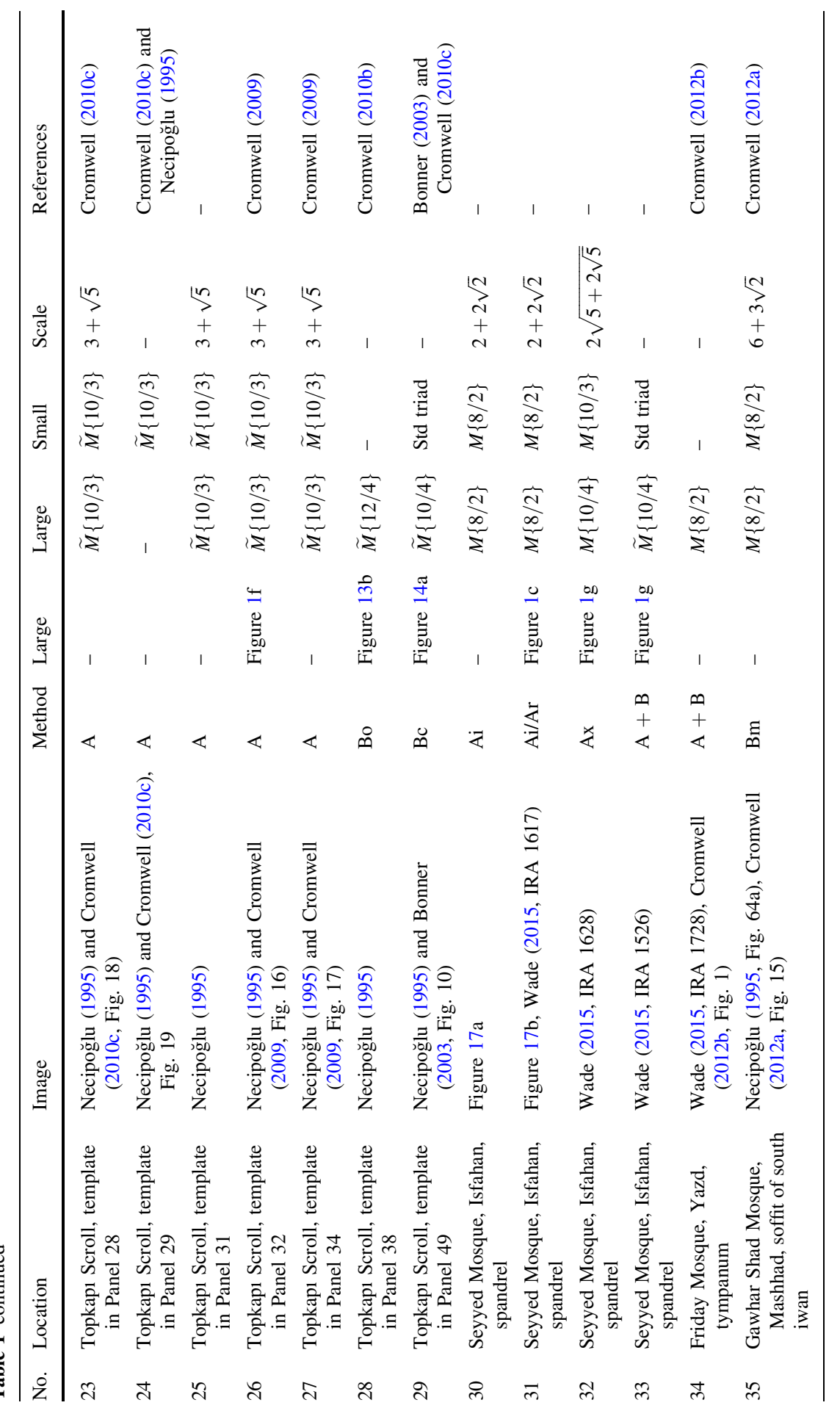




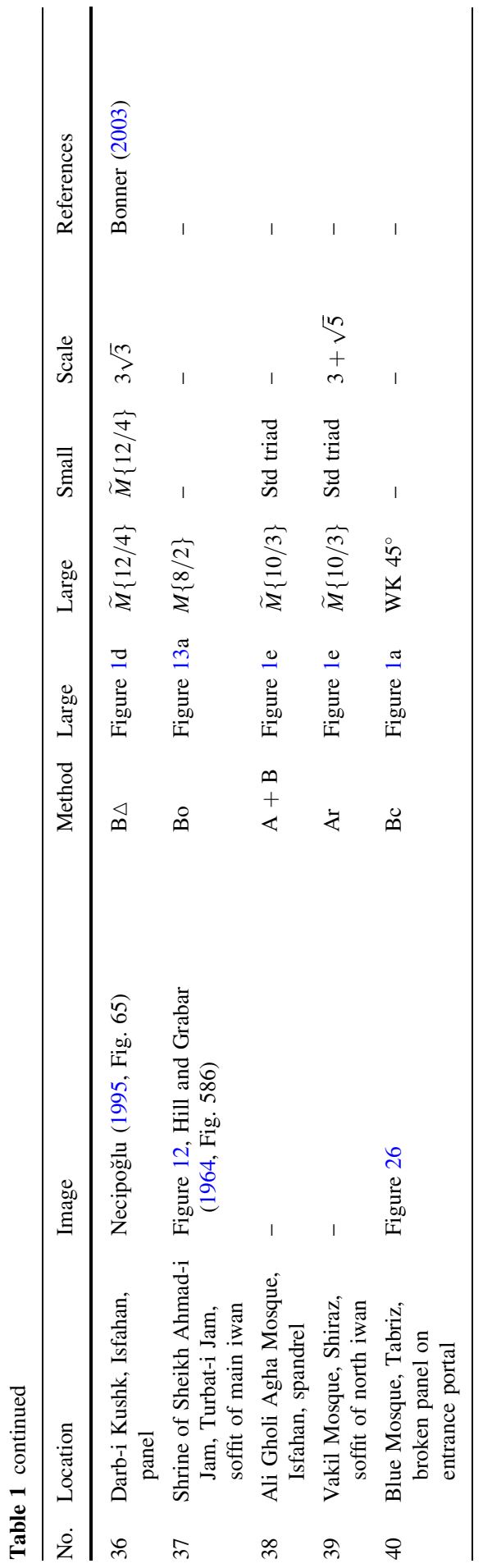




\section{References}

Balmelle, C. and R. Prudhomme. 1985. Le Décor Géometrique de la Mosaïque Romaine: vol 1 Répertoire Graphique et Descriptif des Compositions Linéaires et Isotropes. Picard.

Blair, S. S. and J. M. Bloom. 1995. The Art and Architecture of Islam 1250-1800, Pelican History of Art, Yale Univ. Press.

Bourgoin, J. 1879. Les Eléments de l'Art Arabe: Le Trait des Entrelacs, Firmin-Didot, Paris. Plates reprinted in Arabic Geometric Pattern and Design. Dover Publications, New York, 1973.

Bonner, J. 2003. Three traditions of self-similarity in fourteenth and fifteenth century Islamic geometric ornament. In Proc. ISAMA/Bridges: Mathematical Connections in Art, Music and Science, (Granada, 2003), eds. R. Sarhangi and N. Friedman, 1-12.

Bonner, J. 2015. http://www.bonner-design.com/

Castéra, J.-M. 1996. Arabesques: Art Décoratif au Maroc. ACR Edition.

Castéra, J.-M. 2011. Flying Patterns. In Proc. Bridges: Mathematics, Music, Art, Architecture, (Coimbra, 2011), eds. R. Sarhangi and C. H. Séquin, 263-270.

Cromwell, P. R. 2009. The search for quasi-periodicity in Islamic 5-fold ornament. Math. Intelligencer 31 no 1: 36-56.

Cromwell, P. R. 2010. Hybrid 1-point and 2-point constructions for some Islamic geometric designs. J. Math. and the Arts 4: 21-28.

Cromwell, P. R. 2010. Islamic geometric designs from the Topkapi Scroll I: unusual arrangements of stars. J. Math. and the Arts 4: 73-85.

Cromwell, P. R. 2010. Islamic geometric designs from the Topkapı Scroll II: a modular design system. $J$. Math. and the Arts 4: 119-136.

Cromwell, P. R. 2012a. A modular design system based on the Star and Cross pattern. J. Math. and the Arts 6: 29-42.

Cromwell, P. R. 2012b. Analysis of a multilayered geometric pattern from the Friday Mosque in Yazd. $J$. Math. and the Arts 6: 159-168.

Cromwell, P. R. 2013. On irregular stars in Islamic geometric patterns', preprint.

Cromwell, P. R. 2015. Cognitive bias and claims of quasiperiodicity in traditional Islamic patterns. Math. Intelligencer 37: no 4 30-44.

Cromwell, P. R. and E. Beltrami. 2011. The whirling kites of Isfahan: geometric variations on a theme. Math. Intelligencer 33: no 3 84-93.

Dunbabin, K. M. D. 1999. Mosaics of the Greek and Roman World, Cambridge Univ. Press.

Gailiunas, P. 2011. A recursive construction of a Celtic key pattern. J. Math. and the Arts 5: 105-114.

Golombek, L. and D. Wilber. 1988. The Timurid Architecture of Iran and Turan, Princeton Univ. Press, Princeton.

Hankin, E. H. 1925. The Drawing of Geometric Patterns in Saracenic Art. Memoirs of the Archaeological Society of India, no 15 , Government of India.

Hill, D and O. Grabar. 1964. Islamic Architecture and its Decoration A.D. 800-1500, a Photographic Survey. Faber and Faber, London.

Lee, A. J. 1987. Islamic star patterns. In Muqarnas IV: An Annual on Islamic Art and Architecture. ed. O. Grabar, 182-197, Leiden.

Lu, P. J. and P. J. Steinhardt. 2007. Decagonal and quasi-crystalline tilings in medieval Islamic architecture. Science 315 (23 Feb 2007): 1106-1110.

Makovicky, E. 1992. 800-year old pentagonal tiling from Maragha, Iran, and the new varieties of aperiodic tiling it inspired. In Fivefold Symmetry, ed. I. Hargittai, 67-86, World Scientific.

Michell, G. 2007. The Majesty of Mughul Decoration: The Art and Architecture of Islamic India. Thames and Hudson, London.

Necipoğlu, G. 1995. The Topkap Scroll: Geometry and Ornament in Islamic Architecture. Getty Center Publication, Santa Monica.

Pajares-Ayuela, P. 2001. Cosmatesque Ornament: Flat Polychrome Geometric Patterns in Architecture. W. W. Norton and Co.

Rempel, L. I. 1961. Arkhitekturnie Ornament Uzbekistana (Architectural Ornament in Uzbekistan). Tashkent.

Stierlin, H. 2002. Islamic Art and Architecture from Isfahan to the Taj Mahal. Thames and Hudson, London.

Sutton, D. 2007. Islamic Design: A Genius for Geometry. Wooden Books Ltd, Glastonbury. 
Trilling, J. 2001. The Language of Ornament. Thames and Hudson.

Wade, D. 2015. Pattern in Islamic Art: The Wade Photo-Archive. http://www.patterninislamicart.com/ Wulff, H. E. 1966. The Traditional Crafts of Persia. The M.I.T. Press, Cambridge, Massachusetts. http://www.kufic.info/

Peter Cromwell is a research fellow in the mathematics department at the University of Liverpool. He graduated in mathematics from Warwick University, and obtained his $\mathrm{Ph}$.D. in geometric topology at Liverpool. He has held research fellowships from SERC and the Leverhulme Trust. He is interested in low dimensional geometry, especially problems with a strong visual element. He is the author of Polyhedra (Cambridge University Press, 1999) and Knots and Links (Cambridge University Press, 2004). He has studied geometric ornament for over 20 years, particularly Celtic and Islamic patterns, applying a scientific perspective to some art historical questions. 